\title{
Modeling Antimicrobial Tolerance and Treatment of Heterogeneous Biofilms
}

\author{
Jia Zhao, Paisa Seeluangsawat ${ }^{\dagger}$ and Qi Wang ${ }^{\ddagger}$
}

July 22, 2016

\begin{abstract}
A multiphasic, hydrodynamic model for spatially heterogeneous biofilms based on the phase field formulation is developed and applied to analyze antimicrobial tolerance of biofilms by acknowledging the existence of persistent and susceptible cells in the total population of bacteria. The model implements a new conversion rate between persistent and susceptible cells and its homogeneous dynamics is bench-marked against a known experiment quantitatively. It is then discretized and solved on graphic processing units (GPUs) in 3-D space and time. With the model, biofilm development and antimicrobial treatment of biofilms in a flow cell are investigated numerically. Model predictions agree qualitatively well with available experimental observations. Specifically, numerical results demonstrate that: (i) in a flow cell, nutrient, diffused in solvent and transported by hydrodynamics, has an apparent impact on persister formation, thereby antimicrobial persistence of biofilms; (ii) dosing antimicrobial agents inside biofilms is more effective than dosing through diffusion in solvent; (iii) periodic dosing is less effective in antimicrobial treatment of biofilms in a nutrient deficient environment than in a nutrient sufficient environment. This model provides us with a simulation tool to analyze mechanisms of biofilm tolerance to antimicrobial agents and to derive potentially optimal dosing strategies for biofilm control and treatment.
\end{abstract}

\section{Introduction}

In nature, as soon as bacteria colonize on moisture surfaces, a biofilm is likely to form thereafter, consisting of the micro-organisms aggregated by bacteria along with their self-produced, gluelike exopolysacharride matrix, also known as the extracellular polymeric substance (EPS). It's commonly perceived by the medical community that biofilms are responsible for many diseases or ailments associated with chronic infections, evidenced for example by the survey that biofilms are present in the removed tissue of $80 \%$ of patients undergoing surgery for chronic sinusitis [38]. Unlike a planktonic bacterium, biofilms are hard to be eradicated by the standard antimicrobial treatment [31], which perhaps explains the frequent relapse of chronic diseases or ailments associated with bacterial infections.

Thus, an understanding of the mechanism that underlies biofilm tolerance/persistence to antimicrobial agents can greatly enhance therapeutic treatment of diseases related to biofilms. Intensive

\footnotetext{
*Department of Mathematics, University of South Carolina, Columbia, SC 29208, USA; zhao62@math.sc.edu

${ }^{\dagger}$ Department of Mathematics, University of South Carolina, Columbia, SC 29208, USA; seeluang@math.sc.edu

${ }^{\ddagger}$ Department of Mathematics, University of South Carolina, Columbia, SC 29208, USA; Beijing Computational Science Research Center, Beijing, 100083, China; School of Mathematics, Nankai University, Tianjin 300071, China. qwang@math.sc.edu
} 
research has been conducted, primarily in experiment, to try to understand biofilm structures and dynamics, but the detailed mechanism is still not fully known. Readers may refer to the review papers $[15,31]$ for overviews of current advances in treatment of biofilms. One essential factor for antimicrobial persistence of biofilms is perhaps the existence of persistent cells (persisters) within the biofilm colony, which are consisted of a small portion of dormant bacterial variants that are highly tolerant to antimicrobial agents [4]. Contrasting to persistent cells, the other bacteria are collectively called susceptible bacteria since they respond to antimicrobial treatment sensitively.

From the clinical point of view, understanding the mechanism of persister formation would be useful for biofilm control and eradication, which will impact on treatment of diseases related to biofilms. For reviews on mechanisms underlying the persister formation, readers are referred to the two papers by Kim Lewis [31, 30]. As dormant variants of regular bacterial cells, which don't undergo genetic changes, it is perceived that persisters are converted from regular cells due to stresses [4], such as nutrient depletion [7, 3, 2, 1], existence of antimicrobial agents [34] and so on. Later, when the environment is tolerable, namely nutrient becomes sufficient or the concentration of antimicrobial agents drops under a threshold value, biofilms can relapse [8], which implies that persisters have converted back into susceptible bacteria for regrowth. It is a common belief that persister cells are much slow growers compared to susceptible cells.

Taking into account persister formation, researchers have conducted research on therapeutic treatment of diseases induced by biofilms. The review paper [41] provides some control strategies for biofilms. The dosing strategy when administering antibiotics to treatment of biofilms is also an important issue. There exists an evidence that a concentrated dose of biocide is more effective than using a prolonged dose of a lower concentration [23]. In addition, dosing by shocks is more effective than dosing in a persistent manner [22]. To the best of our knowledge, there have not been any optimal strategies derived for biofilm control or disease treatment related to biofilms so far. Currently, the environmental impact of biocide or side-effect of antibiotics have become common concerns, which makes the development of an optimal antimicrobial strategy even harder.

From the modeling perspective, simple mathematical models have been developed to test certain hypotheses of persister formation based on the experimental evidence that supports the concept of persisters. For instance in [36], the author used a simple mathematical model to show that persister formation can lead to higher bacterial persistence with respect to antimicrobial agents than those grown in planktonic culture. In [24], a 3D agent-based model for biofilm dynamics under antimicrobial treatment was developed, in which it showed that the substrate limitation can contribute to persistence of biofilms with respect to antimicrobial treatment. Notably, Cogan et al. have worked on some possible mechanisms of persister formation using time-dependent, spatially homogeneous models recently $[12,11,29]$.

Some mathematical models on dosing strategies for treating diseases related to biofilms have also been derived. For instance, Cogan et al. discussed effective dosing strategies using a simple mathematical model in $[29,12]$. In [14], he discussed the effect of periodic disinfection using a one-dimensional model. In [44], the adaptive response to dosing protocols for biofilm controls was analyzed, which provided some sufficient conditions for eradicating biofilms using a constant dosing approach. In addition, models analyzing other impact factors, which may contribute to biofilm's persistence to antimicrobial treatment were also proposed. For instance, the author in [17] analyzed and simulated diffusive resistance of bacterial biofilms to penetration of antibiotics.

Most of the modeling efforts in the past focused on reactive kinetics of biofilm persistence and treatment. Very few considered the hydrodynamic effect and the spatio-temporal heterogeneous structures of biofilms in 3D space and time. It is well-known that biofilms are of highly heterogeneous spatial structures and rich temporal dynamics. The spatial heterogeneity can significantly impact on biofilm formation and its function, especially, concerning biofilm's persistence to an- 
timicrobial agents. In this paper, We develop a multiphasic hydrodynamic model for biofilms of multiple bacterial phenotypes; in particular, we limit the phenotypes to the persister and susceptible type. This model extends our previous model of biofilms based on biomass-solvent mixtures [45] by distinguishing between the persister cell and the susceptible cell when biofilms are treated by antimicrobial agents. In this model, the interplay among the various biomass components such as various bacterial types, EPS and solvent is carefully taken into account both hydrodynamically and chemically. The model shows that the dynamical interaction between persistent and susceptible phenotypes can impact dramatically on overall dynamics of the biofilm. It provides the spatiotemporal resolution that is needed for more details about antimicrobial treatment against biofilm colonies in space and time than the previous models can, providing more insight into hydrodynamics of biofilms under antimicrobial treatment.

The paper is organized as follows. In section two, we formulate the hydrodynamic theory for the biofilm system based on the phase field formulation. Then, an efficient numerical solver for the governing partial differential equation system is developed using the semi-implicit finite difference strategy in section three. In section four, numerical results are presented and discussed. Finally, we summarize the result and draw a conclusion.

\section{Mathematical Model Formulation}

We model the biofilm together with its surrounding aqueous environment as a multiphase complex fluid. The biofilm consists of the mixture of biomass and solvent, in which biomass is made up of bacteria of various phenotypes and their products like exopolysaccharide (EPS). Nutrient and antimicrobial agents are small molecule substances dissolved in solvent. Their mass and volume fractions are negligibly small, which will therefore be neglected in this model. However, their chemical effects are important and will therefore be retained. Let $\phi_{b s}$ be the volume fraction of the bacteria that are susceptible to antimicrobial agents, $\phi_{b p}$ the volume fraction of the bacteria that are persistent to the agent, $\phi_{b d}$ the volume fraction of the dead bacteria, $\phi_{b}$ the volume fraction $o$ the bacteria, and $\phi_{p}$ the volume fraction of EPS. We in addition denote the concentration of the nutrient and the antimicrobial agent as $c$ and $d$, respectively, and define $\phi_{n}$ the volume fraction of the biomass, consisting of all the volume fractions for the bacteria as well as EPS,

$$
\phi_{n}=\phi_{p}+\phi_{b s}+\phi_{b p}+\phi_{b d}
$$

In addition to the volume fractions introduced above, the volume fraction of the solvent is denoted as $\phi_{s}$. The incompressibility of the complex fluid mixture then implies

$$
\phi_{s}+\phi_{n}=1 .
$$

To help the reader to better understand the structure of our biofilm model, we show a schematic for the biofilm colony in Figure 1.

In this model, we make a simplifying assumption that all components in the biomass including the bacteria and EPS share the same mass density, which is roughly correct. We denote $\rho_{n}$ and $\rho_{s}$ the density of the biomass and solvent, and $\eta_{n}$ and $\eta_{s}$ the viscosity of the biomass and solvent, respectively. Then, the volume averaged viscosity and density are given respectively by

$$
\eta=\phi_{n} \eta_{n}+\phi_{s} \eta_{s}, \quad \rho=\phi_{n} \rho_{n}+\phi_{s} \rho_{s} .
$$

We assume the bacteria, regardless whether they are live or dead, and the EPS mix with the solvent owing to the osmotic pressure. Then, we adopt the modified free energy introduced in [45] 
This is the modified Flory-Huggins free energy with a conformational entropy, in which $\gamma_{1}$ and $\gamma_{2}$ parametrize the strength of the conformational entropy and the bulk mixing free energy, respectively, $\chi$ is the mixing parameter, $\mathrm{N}$ is the extended polymerization index for the biomass, $k_{B}$ is the Boltzmann constant and $T$ is the absolute temperature.

\subsection{Transport equations for biomass components}

Given the free energy density functional $f$ in equation (4), the 'extended' chemical potentials with respect to biomass components are summarized as follows

$$
\mu_{b s}=\frac{\delta F}{\delta \phi_{b s}}, \quad \mu_{b p}=\frac{\delta F}{\delta \phi_{b p}}, \quad \mu_{b d}=\frac{\delta F}{\delta \phi_{b d}}, \quad \mu_{p}=\frac{\delta F}{\delta \phi_{p}} .
$$

The transport equation for the volume fraction of each biomass component is governed by a reactive Cahn-Hilliard equation,

$$
\partial_{t} \phi_{i}+\nabla \cdot\left(\mathbf{v} \phi_{i}\right)=\nabla \cdot\left(\lambda_{i} \phi_{i} \nabla \mu_{i}\right)+g_{i}, \quad i=b s, b p, b d, p .
$$

where $g_{b s}, g_{b p}, g_{b d}$ and $g_{p}$ are the reactive terms (rates) for the susceptible bacteria, persisters, dead bacteria, and the EPS component, respectively, which will be given in details in the next subsection, $\lambda_{b s}, \lambda_{b p}, \lambda_{b d}$ and $\lambda_{p}$ are the motility parameters for the transport of the four components, respectively, which can be functions of the biomass volume fractions.

\subsection{Transport equations for nutrient and antimicrobial agents}

As mentioned above, the nutrient and antimicrobial agents are treated as phantom materials, in which their volume are completely neglected, but their chemical effects are retained. This is because nutrient and antimicrobial agents all consist of small molecule materials compared to the biomass, which are made up of the bacterial cells and macromolecular EPS. 
In this paper, we denote the concentration of nutrient and antimicrobial agents by $c$ and $d$, respectively. They are assumed to convect with the solvent velocity, to diffuse in the biofilm as well as to react with biomass. The governing equations for the two chemical components are given by the following two reaction-convection-diffusion equations, respectively,

$$
\begin{aligned}
\frac{\partial\left(\phi_{s} c\right)}{\partial t}+\nabla \cdot\left(c \mathbf{v} \phi_{s}\right) & =\nabla \cdot\left(D_{c} \phi_{s} \nabla c\right)+g_{c}, \\
\frac{\partial\left(\phi_{s} d\right)}{\partial t}+\nabla \cdot\left(d \mathbf{v} \phi_{s}\right) & =\nabla \cdot\left(D_{d} \phi_{s} \nabla d\right)+g_{d},
\end{aligned}
$$

where $D_{c}, D_{d}$ are diffusion rates, and $g_{c}, g_{d}$ are the reactive terms for the nutrient and antimicrobial agents, respectively.

\subsection{Hydrodynamic equations for the complex fluid mixture}

For the multiphase complex fluid mixture, the average velocity $\mathbf{v}$ is assumed to be solenoidal. Then, the continuity and the momentum balance equations are given as follows,

$$
\begin{aligned}
\rho\left(\partial_{t} \mathbf{v}+\mathbf{v} \cdot \nabla \mathbf{v}\right) & =-\nabla p+\nabla \cdot \tau-\gamma_{1} k_{B} T \nabla \cdot\left(\nabla \phi_{n} \otimes \nabla \phi_{n}\right), \\
\nabla \cdot \mathbf{v} & =0
\end{aligned}
$$

where $\rho$ is the volume-averaged density, $p$ is the hydrostatic pressure, $\tau$ is the extra stress tensor. The last term in the momentum equation is known as the Ericksen stress [45, 46], which is due to the spatial inhomogeneity of the biomass distribution. The Ericksen stress arises wherever there exists a density/concentration/volume fraction gradients in the mixture, which also contributes to the osmotic pressure of the system. In this paper, we are interested in growth dynamics of the biofilm, whose time scale is significantly larger than the relaxation time scale in the EPS. We therefore model all effective biofilm components as viscous fluids, namely, $\tau=2 \eta \mathbf{D}$, where $\mathbf{D}=\frac{1}{2}\left(\nabla \mathbf{v}+\nabla \mathbf{v}^{T}\right)$ is the rate of deformation tensor. We note that the viscoelastic effect is important only when we investigate biofilm dynamics in shorter time scales.

\subsection{Reactive kinetics}

In order to complete the model formulation, we need to derive reactive kinetics for every effective biomass and chemical component. We develop reactive kinetics as general as possible by taking into account all experimental evidences and hypotheses in the literature. Later in the numerical study section, we focus on a specific model with parameter values collected from the literature and our best guesses.

Bacterial growth is fueled by the nutrient and inhibited by antimicrobial agents present in biofilms. The two different bacterial phenotypes (the susceptible and persister cells) have different growth mechanisms; thus we assume that both the susceptible and the persister cell grow on their own at their own rates. The growth of persisters are perceived to be much slower that that of susceptible cells. For both bacterial phenotypes, death rates due to natural causes are taken into account as well. In addition, both susceptible bacteria and persisters can be killed by antimicrobial agents, but persisters are killed in a much slower rate due to its antimicrobial persistence. We allow all these features in the model so that they can be turned on and off depending on the time scale that we are interested in. It is perceived that susceptible bacteria and persisters can be converted mutually, based on the stage of their growth [31] and the environment [12], such as accessibility to nutrient and exposure to antimicrobial agents. 
Based on the mechanisms mentioned above, reactive kinetics for the two types of live bacteria are proposed as follows:

$$
\begin{aligned}
& g_{b s}=\frac{C_{2} c}{K_{1}+c}\left(1-\frac{\phi_{b s}}{\phi_{b s 0}}\right) \phi_{b s}-b_{s p} \phi_{b s}+b_{p s} \phi_{b p}-\left(\frac{r_{b s} K_{s d}^{2}}{K_{s d}^{2}+c^{2}}+\frac{C_{3} d}{K_{3}+d}\right) \phi_{b s} \\
& g_{b p}=\frac{C_{4} c}{K_{2}+c}\left(1-\frac{\phi_{b p}}{\phi_{b p 0}}\right) \phi_{b p}+b_{s p} \phi_{b s}-b_{p s} \phi_{b p}-\left(\frac{r_{b p} K_{p d}^{2}}{K_{p d}^{2}+c^{2}}+\frac{C_{12} d}{K_{3}+d}\right) \phi_{b p},
\end{aligned}
$$

where $C_{2}$ and $C_{4}$ are growth rates for the susceptible and the persister, respectively; $\phi_{b s 0}$ and $\phi_{b p 0}$ are carrying capacities for susceptible bacteria and persisters due to growth, respectively; $b_{s p}$ and $b_{p s}$ are conversion rates between the two types of bacteria; $r_{b s}$ and $r_{b p}$ are natural death rates due to nutrient depletion for susceptible bacteria and persisters, respectively, and $C_{3}$ and $C_{12}$ are death rates for susceptible bacteria and persisters due to antimicrobial agents, respectively. $K_{1}, K_{2}, K_{3}$, $K_{s d}, K_{p d}$ are half saturation constants in the Monod models adopted. In this model, the decay rates of the live bacteria increase as the concentration of the antimicrobial agents increase while decrease with respect to the concentration of nutrient. Based on the experimental evidence, we assume $r_{b s}>>r_{b p}, C_{2}>>C_{4}$ and $C_{3}>>C_{12}$.

In some studies [3, 21], $b_{s p}$ and $b_{p s}$ are assumed constants. In other studies [12], nonlinear conversion rates were used

$$
b_{s p}=b_{s p 1} \frac{c}{c+k_{s p}}, \quad b_{p s}=b_{p s 1}\left(1-\frac{1}{1+e^{-\frac{d-d_{0}}{\varepsilon}}}\right),
$$

where $b_{s p 1}$ and $b_{p s 1}$ are the maximum conversion rates and $d_{0}$ is a threshold value for antimicrobial agents, $\varepsilon$ is a small parameter. These nonlinear functions improved the model prediction to some extent. But, the improvement is still not satisfactory.

Here, we propose a new formulation for the switch function $b_{s p}$ and $b_{p s}$ between the two types of bacteria, based on the experimental evidence as well as latest interpretation about the conversion in the literature. We assume conversion between the susceptible and the persister cell is primarily by the "stress" [34], which includes two parts: the stress from exogenous sources, such as antimicrobial agents, and the self-imposed stress, such as starvation. Based on this, we propose the conversion rates $b_{s p}$ and $b_{p s}$ as functions of concentrations of both nutrient and antimicrobial agents,

$$
b_{s p}=b_{s p}(c, d), \quad b_{p s}=b_{p s}(c, d) .
$$

For $b_{s p}$, we note that there should be two separate leading order terms, which represent the effect of nutrient and antimicrobial agents, respectively. For instance, nutrient depletion alone can induce persister formation $[32,7,8]$; without nutrient depletion, persister formation can also be induced in response to the antimicrobial stress [19]. Thus, we propose the conversion rate $b_{s p}$ as follows:

$$
b_{s p}=\left(b_{s p 1} \frac{k_{s p c}^{2}}{k_{s p c}^{2}+c^{2}}+b_{s p 2} \frac{d^{2}}{k_{s p d}^{2}+d^{2}}\right)\left(1-\frac{\phi_{b p}}{\phi_{b p 0}}\right),
$$

where the first term in the first multiplicative factor describes the mechanism that bacteria turn into persisters due to nutrient depletion, the second term in the factor depicts the 'stress' induced conversion by antimicrobial agents, and the second multiplicative factor represents the carrying capacity for the persister to show the conversion rate is limited with an upper bound $\phi_{b p 0}$. Here $b_{s p 1}$ and $b_{s p 2}$ represent the maximum conversion rates from the susceptible to the persister due to nutrient depletion and antimicrobial stress, respectively. In the formula, we use a monod function to model the transitional effect. Although it is plausible to propose one more term, which depends 
both on nutrient and antimicrobial agents, this correlation is perceived as a high-order effect and is therefore omitted for simplicity. The second multiplicative factor limits the conversion to the carrying capacity of persister cells, beyond which there would be no more conversion going on.

For $b_{p s}$, it is assumed nonzero only if both nutrient depletion and the antimicrobial stress are under certain threshold values. Namely, we expect it to possess the following properties: it is close to zero when the level of antimicrobial agents is high enough, since a biofilm with persisters is tolerable to antimicrobial treatment, and monotonically increases to a constant level as the concentration of antimicrobial agents drops below a threshold value, since biofilms are observed to recover after antimicrobial treatment. In addition, the availability of nutrient should facilitate the conversion process [8]. Based on these requirements, we propose the following conversion rate:

$$
b_{p s}=b_{p s 1} \frac{c^{2}}{k_{p s c}^{2}+c^{2}} \frac{k_{p s d}^{2}}{k_{p s d}^{2}+d^{2}},
$$

where $b_{p s 1}$ is the maximum conversion rate, $k_{p s c}$ and $k_{p s d}$ are two half-saturation constants.

Besides the live bacteria, the volume fraction of the dead bacteria is also tracked in this model. It is assumed that the dead bacteria stay within the biofilm in the time scale that this model is considered. Meanwhile, we assume some dead bacterial cells are attached to the biofilm acting as EPS while others are converted into solvent due to cell lysis [5] at certain specified rates in the time scale of our interest,

$$
g_{b d}=\left(\frac{r_{b s} K_{s d}^{2}}{K_{s d}^{2}+c^{2}}+\frac{C_{3} d}{K_{3}+d}\right) \phi_{b s}+\left(\frac{r_{b p} K_{p d}^{2}}{K_{p d}^{2}+c^{2}}+\frac{C_{12} d}{K_{3}+d}\right) \phi_{b p}-r_{d p} \phi_{b d}-r_{b d} \phi_{b d},
$$

where $r_{b s}, r_{b p}$ are the natural death rates, $C_{3}, C_{12}$ are the antimicrobial killing rates, given before, $r_{d p}$ represents the conversion rate from dead bacteria to EPS, and $r_{b d}$ is the cell lysis rate of the decomposition of dead bacteria into solvent.

EPS is produced by susceptible bacteria and persisters at different rates as well as converted from dead bacteria. Over times, EPS can be dissolved in a slow rate into solvent. So, the reactive kinetics for EPS is proposed as follows

$$
g_{p}=\left(\frac{C_{5} c}{K_{5}+c} \phi_{b s}+\frac{C_{6} c}{K_{6}+c} \phi_{b p}\right)\left(1-\frac{\phi_{p}}{\phi_{p 0}}\right)+r_{d p} \phi_{b d}-r_{p} \phi_{p},
$$

where $C_{5}, C_{6}$ are the growth rate of EPS due to susceptible bacteria and persisters, respectively, $K_{5}, K_{6}$ are half saturation constants, $\phi_{p 0}$ is the maximum volume fraction that EPS is produced by live cells in the biofilm, and $r_{p}$ represents the dissolving rate of EPS into solvent.

We assume the nutrient is consumed by the live bacteria only and the nutrient decay rate is proportional to the bacterial volume fraction:

$$
g_{c}=-C_{7}\left(\phi_{b s}+\phi_{b p}\right) \frac{c}{K_{7}+c}
$$

where $K_{7}$ is a saturation constant and $C_{7}$ parametrizes the consumption rate of nutrient.

The concentration of antimicrobial agents depends on concentrations of live bacteria and EPS. It is 'absorbed/consumed' by the live bacteria and possibly diluted by the EPS and dead bacteria via chemical reactions. Thus, the presence of all bacteria and EPS can reduce the concentration of antimicrobial agents. We thus propose the decay rate of antimicrobial agents as follows:

$$
g_{d}=-\left(C_{8} \phi_{b s}+C_{9} \phi_{b p}+C_{10} \phi_{b d}+C_{11} \phi_{p}\right) \frac{d}{K_{8}+d},
$$

where $K_{8}$ is a half saturation constant, $C_{8}, C_{9}, C_{10}$ and $C_{11}$ are the decay rates of antimicrobial agents due to the drug-susceptible bacteria interaction, drug-persister cell interaction, drug-dead bacteria interaction and drug-EPS interaction, respectively. 


\subsection{Boundary Conditions}

In this study, we assume that the biofilm is confined in a cubic domain in $3 \mathrm{D}$ space: $\left[0, L_{x}\right] \times$ $\left[0, L_{y}\right] \times\left[\begin{array}{ll}0, & L_{z}\end{array}\right]$ where $L_{x}, L_{y}, L_{z}$ are the length in the $\mathrm{x}, \mathrm{y}$, and $\mathrm{z}$ direction, respectively. The boundary conditions for the model are given based on the physical settings that we intend to simulate. In this paper, we consider two physical settings. One is for a quiescent aqueous long channel and the other is for a short water tube or flow cell with a rectangular cross-section, where flows can flow in and out of the domain.

To mimic the biofilm development in a long channel, both $\mathrm{x}$ and $\mathrm{z}$ directions are assumed periodic. In the y direction, zero wall velocity for $\mathbf{v}$ and no-flux boundary conditions are imposed for concentrations/volume fractions of biomass, nutrient and antimicrobial agents, respectively,

$$
\begin{aligned}
\left.\mathbf{v}\right|_{y=0, L_{y}} & =0 \\
{\left.\left[c \mathbf{v} \phi_{s}-D_{c} \phi_{s} \nabla c\right] \cdot \mathbf{n}\right|_{y=0, L_{y}} } & =0 \\
{\left.\left[d \mathbf{v} \phi_{s}-D_{d} \phi_{s} \nabla d\right] \cdot \mathbf{n}\right|_{y=0, L_{y}} } & =0, \\
\left.\nabla \phi_{i} \cdot \mathbf{n}\right|_{y=0, L_{y}} & =0, \quad i=b s, b p, b d, p, \\
\left.\left(\mathbf{v} \phi_{i}-\lambda_{i} \phi_{i} \nabla \frac{\delta F}{\delta \phi_{i}}\right) \cdot \mathbf{n}\right|_{y=0, L_{y}} & =0, \quad i=b s, b p, b d, p .
\end{aligned}
$$

We can also impose a nutrient feeding condition $\left.c\right|_{y=L_{y}}=c^{*}$ in place of the zero-flux condition in certain parts of the boundary if needed.

For the case of biofilms in a short flow cell, periodic boundary conditions are imposed only in the $\mathrm{z}$ direction. The $\mathrm{x}$ axis is assumed to align with the inlet-outlet direction. The inlet velocity at $x=0$ is given by

$$
\mathbf{v}_{0}=\left(p_{0} y(1-y), 0,0\right),
$$

where $p_{0}$ is a prescribed pressure gradient. By assuming that the solvent has already reached a steady state while flowing out of the cell at $x=L_{x}$, we prescribe $\frac{\partial \mathbf{v}}{\partial x}=0$ at the outlet end. For the nutrient concentration $c$, we impose the feeding boundary condition at $x=0$ as $c=c_{0}(y)$ and flux-free condition $c_{x}=0$ is assumed at $x=L_{x}$. For the biomass components, we impose no-flux boundary conditions in the $\mathrm{x}$ direction,

$$
\begin{aligned}
\left.\nabla \phi_{i} \cdot \mathbf{n}\right|_{x=0, L_{x}} & =0, \quad i=b s, b p, b d, p \\
\left.\left(\mathbf{v} \phi_{i}-\lambda_{i} \phi_{i} \nabla \frac{\delta F}{\delta \phi_{i}}\right) \cdot \mathbf{n}\right|_{x=0, L_{x}} & =0, \quad i=b s, b p, p, b d .
\end{aligned}
$$

We remark that this boundary condition works for a limited time frame before the biomass reaches the boundary. Beyond that moment, it must be modified. In the y direction, we impose no-flux boundary conditions for nutrient, antimicrobial agents, and biomass components, respectively, same as equation (19).

\subsection{A reduced model and nondimensionalization}

In the development of reactive kinetics, we have proposed a general model by taking into account many experimental evidences and theoretical hypotheses, which involves many time scales. In the study to follow, we drop the terms which have weak effects on biofilm dynamics within the time scale that we're interested in. Specifically, we set

$$
C_{4}=C_{12}=r_{b p}=r_{d p}=r_{b d}=r_{p}=0
$$


This means that we consider the persisters do not growth on their own fueled by nutrient nor they are killed by antimicrobial agents; dead bacteria can't be converted into EPS nor solvent in the time scale that we consider; and EPS does not decay into solvent.

Since most of the model parameters are not directly measurable by experiments and their specific functional form reflect the trend of the reaction, we set some of the reactive rates and half-saturation rate the same based on our best guesses,

$$
C_{8}=C_{9}=C_{10}=C_{11}, K_{i}=K_{s p d}=K_{s p c}=K_{p s c}=K_{p s d}, 1 \leq i \leq 8 .
$$

We believe that this choice of parameters may affect the model prediction quantitatively, but not qualitatively.

Let $t_{0}$ and $h$ represent the reference time and length scale in the biofilm system. We use these two characteristic scales to nondimensionalize the variables and equations. In this study, we assume each biofilm component has the same constant mobility, denoted by $\lambda$. The dimensionless variables are then given by

$$
\begin{gathered}
\tilde{t}=\frac{t}{t_{0}}, \tilde{x}=\frac{x}{h}, \tilde{\mathbf{v}}=\frac{\mathbf{v} t_{0}}{h}, \tilde{\tau}=\frac{\tau t_{0}^{2}}{\rho_{0} h^{2}}, \tilde{p}=\frac{p t_{0}^{2}}{\rho_{0} h^{2}}, \tilde{c}=\frac{c}{c_{0}}, \tilde{d}=\frac{d}{d_{0}}, \\
\Lambda=\frac{\lambda \rho_{0}}{t_{0}}, \quad R e_{i}=\frac{\rho_{0} h^{2}}{\eta_{i} t_{0}}, i=s, b, p . \quad \Gamma_{1}=\frac{\gamma_{1} k T t_{0}^{2}}{\rho_{0} h^{4}}, \quad \Gamma_{2}=\frac{\gamma_{2} k T t_{0}^{2}}{\rho_{0} h^{2}}, \quad \tilde{\rho}=\phi_{s} \frac{\rho_{s}}{\rho_{0}}+\phi_{n} \frac{\rho_{n}}{\rho_{0}}, \\
\tilde{D}_{c}=\frac{D_{c} t_{0}}{h^{2}}, \quad \tilde{D}_{d}=\frac{D_{d} t_{0}}{h^{2}}, \tilde{C}_{i}=C_{i} t_{0}, i=2,3,5,6,7,8 ; \tilde{b}_{i}=b_{i} t_{0}, i=s p 1, s p 2, p s 0 \\
\tilde{K}_{i}=\frac{K_{i}}{c_{0}}, i=1, s p c, p s c ; \tilde{K}_{j}=\frac{K_{j}}{d_{0}}, j=3,8, s p d, p s d ; \tilde{r}_{i}=r_{i} t_{0}, i=b s, b d .
\end{gathered}
$$

where $c_{0}, d_{0}$ denote the characteristic concentration of nutrient and antimicrobial agents, respectively.

For simplicity, we drop the tilde ${ }^{\sim}$ on the dimensionless variables and parameters. The nondimensionalized PDEs governing the biofilm system are summarized as follows:

$$
\left\{\begin{aligned}
\rho\left(\frac{\partial \mathbf{v}}{\partial t}+\mathbf{v} \cdot \nabla \mathbf{v}\right) & =\nabla \cdot(2 \eta \mathbf{D})-\nabla p-\Gamma_{1} \nabla \cdot\left(\nabla \phi_{n} \otimes \nabla \phi_{n}\right), \\
\nabla \cdot \mathbf{v} & =0 \\
\frac{\partial}{\partial t} \phi_{i}+\nabla \cdot\left(\phi_{i} \mathbf{v}\right) & =\nabla \cdot\left(\Lambda \phi_{i} \nabla \frac{\delta F}{\delta \phi_{i}}\right)+g_{i}, \quad i=b s, b p, b d, p, \\
\frac{\partial \phi_{s} c}{\partial t}+\nabla \cdot\left(c \mathbf{v} \phi_{s}\right) & =\nabla \cdot\left(D_{c} \phi_{s} \nabla c\right)+g_{c}, \\
\frac{\partial \phi_{s} d}{\partial t}+\nabla \cdot\left(d \mathbf{v} \phi_{s}\right) & =\nabla \cdot\left(D_{d} \phi_{s} \nabla d\right)+g_{d},
\end{aligned}\right.
$$

where $\eta=\frac{\phi_{b}}{R e_{b}}+\frac{\phi_{s}}{R e_{s}}+\frac{\phi_{p}}{R e_{p}}$, and the reactive terms for the component are given by

$$
\begin{aligned}
g_{b s} & =\frac{C_{2} c}{K_{1}+c}\left(1-\frac{\phi_{b s}}{\phi_{b s 0}}\right) \phi_{b s}-b_{s p} \phi_{b s}+b_{p s} \phi_{b p}-\left(\frac{r_{b s} K_{s d}^{2}}{K_{s d}^{2}+c}+\frac{C_{3} d}{K_{3}+d}\right) \phi_{b s} \\
g_{b p} & =b_{s p} \phi_{b s}-b_{p s} \phi_{b p} \\
g_{b d} & =\left(r_{b s} \frac{K_{s d}^{2}}{K_{s d}^{2}+c^{2}}+\frac{C_{3} d}{K_{3}+d}\right) \phi_{b s}-r_{b d} \phi_{b d} \\
g_{p} & =\left[\frac{C_{5} c}{K_{1}+c} \phi_{b s}+\frac{C_{6} c}{K_{1}+c} \phi_{b p}\right]\left(1-\frac{\phi_{p}}{\phi_{p 0}}\right) \\
g_{c} & =-\left(\phi_{b s}+\phi_{b p}\right) \frac{C_{7} c}{K_{1}+c} \\
g_{d} & =-C_{8} \phi_{n} \frac{d}{K_{3}+d}
\end{aligned}
$$


with the conversion rates

$$
\begin{aligned}
& b_{s p}=\left(b_{s p 1} \frac{k_{s p c}^{2}}{k_{s p c}^{2}+c^{2}}+b_{s p 2} \frac{d^{2}}{k_{s p d}^{2}+d^{2}}\right)\left(1-\frac{\phi_{b p}}{\phi_{b p 0}}\right), \\
& b_{p s}=b_{p s 1 \frac{c^{2}}{k_{p s c}^{2}+c^{2}} \frac{k_{p s d}^{2}}{k_{p s d}^{2}+d^{2}} .}
\end{aligned}
$$

Remark: By ignoring spatial effects, the model reduces to a system of coupled ordinary differential equations for a spatially homogeneous (bulk) biofilm system. It is then solved using a Matlab ODE solver. For the inhomogeneous biofilm equations, we will develop a new numerical method to solve them next.

\section{Numerical schemes and GPU implementation}

We develop a second order finite difference scheme to solve the coupled partial differential equations in the biofilm model and then implement it in a CPU-GPU hybrid environment. In the following, we denote the extrapolated data using over-lines; for instance, $\overline{\mathbf{v}}^{n+1}=2 \mathbf{v}^{n}-\mathbf{v}^{n-1}$. We first present the 2 nd order finite difference scheme.

\subsection{A second-order semi-discrete numerical scheme}

Given the initial condition $\left(\mathbf{v}^{0}=0, s^{0}=p^{0}=0, \phi_{b s}^{0}, \phi_{b p}^{0}, \phi_{b d}^{0}, \phi_{p}^{0}, c^{0}, d^{0}\right)$, we first compute $\left(\mathbf{v}^{1}\right.$, $\left.s^{1}, p^{1}, \phi_{b s}^{1}, \phi_{b p}^{1}, \phi_{b d}^{1}, c^{1}, d^{1}\right)$ by a first-order scheme. Having computed $\left(\mathbf{v}^{n-1}, s^{n-1}, p^{n-1}, \phi_{b s}^{n-1}\right.$, $\left.\phi_{b p}^{n-1}, \phi_{b d}^{n-1}, \phi_{p}^{n-1}, c^{n-1}, d^{n-1}\right)$ and $\left(\mathbf{v}^{n}, s^{n}, p^{n}, \phi_{b s}^{n}, \phi_{b p}^{n}, \phi_{b d}^{n}, \phi_{p}^{n}, c^{n}, d^{n}\right)$, where $n \geq 2$, we calculate $\left(\mathbf{v}^{n+1}, s^{n+1}, p^{n+1}, \phi_{b s}^{n+1}, \phi_{b p}^{n+1}, \phi_{b d}^{n+1}, \phi_{p}^{n+1}, c^{n+1}, d^{n+1}\right)$ in the following steps,

1. Update $\rho^{n+1}$ and $\eta^{n+1}$ :

$$
\begin{aligned}
\rho^{n+1} & =\left(\bar{\phi}_{b s}^{n+1}+\bar{\phi}_{b p}^{n+1}+\bar{\phi}_{b d}^{n+1}+\bar{\phi}_{p}^{n+1}\right) \rho_{n}+\bar{\phi}_{s}^{n+1} \rho_{s}, \\
\eta^{n+1} & =\left(\bar{\phi}_{b s}^{n+1}+\bar{\phi}_{b p}^{n+1}+\bar{\phi}_{b d}^{n+1}+\bar{\phi}_{p}^{n+1}\right) \eta_{n}+\bar{\phi}_{s}^{n+1} \eta_{s} .
\end{aligned}
$$

2. Solve $\mathbf{u}^{n+1}$ as the solution of

$$
\left\{\begin{array}{l}
\rho^{n+1} \frac{3 \mathbf{u}^{n+1}-4 \mathbf{v}^{n}+\mathbf{v}^{n-1}}{2 \delta t}+\rho^{n+1} \overline{\mathbf{v}}^{n+1} \cdot \nabla \overline{\mathbf{v}}^{n+1}+\frac{1}{2}\left(\nabla \cdot\left(\rho^{n+1} \overline{\mathbf{v}}^{n+1}\right)\right) \overline{\mathbf{v}}^{n+1}+\frac{1}{R e_{a}} \nabla s^{n}+\nabla p^{n} \\
-\frac{1}{R e_{a}} \nabla^{2} \mathbf{u}^{n+1}=-\Gamma_{1} \nabla^{2} \bar{\phi}_{n}^{n+1} \nabla \bar{\phi}_{n}^{n+1}+\nabla \cdot\left(\eta^{n+1}\left(\nabla \overline{\mathbf{v}}^{n+1}+\left(\nabla \overline{\mathbf{v}}^{n+1}\right)^{T}\right)\right)-\frac{1}{R e_{a}} \nabla^{2} \overline{\mathbf{v}}^{n+1}, \\
\left.\mathbf{u}^{n+1}\right|_{y=0, L_{y}}=0,
\end{array}\right.
$$

where $R e_{a}$ is the averaged Reynolds number, computed by $\frac{1}{R e_{a}}=\frac{\phi_{b, \max }}{R e_{b}}+\frac{\phi_{p, \max }}{R e_{p}}+\frac{1-\phi_{b, \max }-\phi_{p, \max }}{R e_{s}}$, $R e_{b}, R e_{p}$ and $R e_{s}$ are the Reynolds numbers for bacteria, EPS and solvent, respectively, and $\phi_{i, \max }=\max _{\mathbf{x} \in \Omega} \phi_{i}(\mathbf{x}), \quad i=b, p$.

3. Solve intermediate variable $\psi^{n+1}$ as the solution of

$$
\left\{\begin{array}{rlr}
-\nabla \cdot\left(\frac{1}{\rho^{n+1}} \nabla \psi^{n+1}\right) & = & \nabla \cdot \mathbf{u}^{n+1} \\
\left.\frac{\partial \psi^{n+1}}{\partial n}\right|_{y=0, L_{y}} & = & 0 .
\end{array}\right.
$$


4. Update $\left(\mathbf{v}^{n+1}, s^{n+1}, p^{n+1}\right)$ :

$$
\left\{\begin{array}{l}
\mathbf{v}^{n+1}=\mathbf{u}^{n+1}+\rho^{n+1} \nabla \psi^{n+1}, \\
s^{n+1}=s^{n}-\nabla \cdot \mathbf{u}^{n+1}, \\
p^{n+1}=p^{n}-\frac{3 \psi^{n+1}}{2 \delta t}+\frac{1}{R e_{a}} s^{n+1} .
\end{array}\right.
$$

5. Update volume fractions of biomass $\left(\phi_{b s}^{n+1}, \phi_{b p}^{n+1}, \phi_{b d}^{n+1}, \phi_{p}^{n+1}\right)$ :

$$
\left\{\begin{array}{l}
\frac{3 \phi_{b s}^{n+1}-4 \phi_{b s}^{n}+\phi_{b s}^{n-1}}{2 \delta t}+\nabla \cdot\left(\bar{\phi}_{b s}^{n+1} \mathbf{v}^{n+1}\right)=\Lambda \nabla \cdot\left(\bar{\phi}_{b s}^{n+1} \mathcal{F}\left(\phi_{b s}^{n+1}+{\overline{\phi_{b p}+\phi_{b d}+\phi_{p}}}^{n+1}\right)\right)+g_{b s}^{n+1}, \\
\frac{3 \phi_{b p}^{n+1}-4 \phi_{b p}^{n}+\phi_{b p}^{n-1}}{2 \delta t}+\nabla \cdot\left(\bar{\phi}_{b p}^{n+1} \mathbf{v}^{n+1}\right)=\Lambda \nabla \cdot\left(\bar{\phi}_{b p}^{n+1} \mathcal{F}\left(\phi_{b p}^{n+1}+{\overline{\phi_{b s}+\phi_{b d}+\phi_{p}}}^{n+1}\right)\right)+g_{b p}^{n+1}, \\
\frac{3 \phi_{b d}^{n+1}-4 \phi_{b d}^{n}+\phi_{b d}^{n-1}}{2 \delta t}+\nabla \cdot\left(\bar{\phi}_{b d}^{n+1} \mathbf{v}^{n+1}\right)=\Lambda \nabla \cdot\left(\bar{\phi}_{b d}^{n+1} \mathcal{F}\left(\phi_{b d}^{n+1}+{\overline{\phi_{b p}+\phi_{p}+\phi_{b s}}}^{n+1}\right)\right)+g_{b d}^{n+1}, \\
\frac{3 \phi_{p}^{n+1}-4 \phi_{p}^{n}+\phi_{p}^{n-1}}{2 \delta t}+\nabla \cdot\left(\bar{\phi}_{p}^{n+1} \mathbf{v}^{n+1}\right)=\Lambda \nabla \cdot\left(\bar{\phi}_{p}^{n+1} \mathcal{F}\left(\phi_{p}^{n+1}+{\overline{\phi_{b p}+\phi_{b d}+\phi_{b s}}}^{n+1}\right)\right)+g_{p}^{n+1},
\end{array}\right.
$$

where $\mathcal{F}^{n+1}(\phi)=\left(\frac{1}{N} \frac{1}{\bar{\phi}_{n}^{n+1}+\varepsilon}+\frac{1}{1-\bar{\phi}_{n}^{n+1}}-2 \chi\right) \Gamma_{2} \nabla \phi-\Gamma_{1} \nabla \nabla^{2} \phi$, and the reactive terms are discretized by

$$
\begin{aligned}
g_{b s 1}^{n+1} & =\frac{C_{2} \bar{c}^{n+1}}{K_{2}+\bar{c}^{n+1}}\left(1-\frac{\bar{\phi}_{b s}^{n+1}}{\phi_{b s 0}}\right) \phi_{b s}^{n+1}-\bar{b}_{s p}^{n+1} \phi_{b s}^{n+1}+\bar{b}_{p s}^{n+1} \bar{\phi}_{b p}^{n+1}-\left(\frac{r_{b s} K_{s d}^{2}}{K_{s d}^{2}+\left(\bar{c}^{n+1}\right)^{2}}+\frac{C_{3} \bar{d}^{n+1}}{K_{3}+\bar{d}^{n+1}}\right) \phi_{b s}^{n+1}, \\
g_{b p 1}^{n+1} & =-\bar{b}_{p s}^{n+1} \phi_{b p}^{n+1}+\bar{b}_{s p}^{n+1} \bar{\phi}_{b s}^{n+1}, \\
g_{b d}^{n+1} & =\left(r_{b s} \frac{K_{s d}^{2}}{K_{s d}^{2}+\left(\bar{c}^{n+1}\right)^{2}}+\frac{C_{3} \bar{d}^{n+1}}{K_{3}+\bar{d}^{n+1}}\right) \bar{\phi}_{b s}^{n+1}-r_{b d} \phi_{b d}^{n+1} \\
g_{p}^{n+1} & =\left(\frac{C_{5} \bar{c}^{n+1}}{K_{5}+\bar{c}^{n+1}} \bar{\phi}_{b s}^{n+1}+\frac{C_{6} \bar{c}^{n+1}}{K_{6}+\bar{c}^{n+1}} \bar{\phi}_{b p}^{n+1}\right)\left(1-\frac{\phi_{p}^{n+1}}{\phi_{p 0}}\right) .
\end{aligned}
$$

6. Update functional components $\left(c^{n+1}, d^{n+1}\right)$ :

$$
\left\{\begin{array}{c}
\frac{3 \phi_{s}^{n+1} c^{n+1}-4 \phi_{s}^{n} c^{n}+\phi_{s}^{n-1} c^{n-1}}{2 \delta t}+\mathbf{v}^{n+1} \cdot \nabla\left(c^{n+1} \phi_{s}^{n+1}\right) \\
=\nabla \cdot\left(D_{c} \phi_{s}^{n+1} \nabla c^{n+1}\right)-\left(\phi_{b s}^{n+1}+\phi_{b p}^{n+1}\right) \frac{C_{7} c^{n+1}}{K_{7}+c^{n}} \\
\frac{3 \phi_{s}^{n+1} d^{n+1}-4 \phi_{s}^{n} d^{n}+\phi_{s}^{n-1} d^{n-1}}{2 \delta t}+\mathbf{v}^{n+1} \cdot \nabla\left(d^{n+1} \phi_{s}^{n+1}\right) \\
=\nabla \cdot\left(D_{d} \phi_{s}^{n+1} \nabla d^{n+1}\right)-C_{8} \phi_{n}^{n+1} \frac{d^{n+1}}{K_{8}+d^{n}}
\end{array}\right.
$$

Here are several remarks regarding this numerical scheme.

Remark 3.1 Here we introduced a stabilizer term $-\frac{1}{R e_{a}} \nabla^{2} \mathbf{v}$ on both side of (29) [40, 47], treating one term implicitly and the other explicitly via extrapolation. The stabilizer introduces a $O\left(\delta t^{2}\right)$ error, which is consistent with our second-order scheme.

Remark 3.2 A Gauge-Uzawa method [35] is used to solve the Navier-Stokes equation with s and $\psi$ as intermediate variables. Gauge-Uzawa method lies in the category of projection method, where an intermediate variable $\mathbf{u}$ is solved which satisfies the boundary condition, but not the divergence free constraint. Then a project step is carried out to adjust $\mathbf{u}$ to obey the divergence free constraint. 
The scheme above is presented as semi-discrete in time. Second order central finite difference with stagged grids is used to discrete the equation system in space, where the velocity field $\mathbf{v}$ is discretized on the surface of each grid, with the pressure $p$, the phase variables $\phi_{b s}, \phi_{b p}, \phi_{b d}, \phi_{p}$ and variables $c, d$ being discretized on the center of the grid $[48,49,20]$. The resulting algebraic system is implemented on a GPU using CUDA for high performance computing. We use a preconditioned BiCG solver (using the package cusp [6]) to solve the sparse linear system generated from the full discretization, where the preconditioner is constructed as the inverse of a good approximation of the implicit operator by approximating the variable coefficient with a constant coefficient [49]. The preconditioner can then be computed by the Fast Fourier Transform. The data are saved as HDF5 format, which can be visualized using VisIt [10]. Currently, one solver can deal with up to $256^{3}$ meshes, limited by the global memory size of a single GPU. The scheme is second order in space and time in theory, which is confirmed by numerical convergence tests.

\section{Numerical Results and Discussion}

\subsection{Model parameters and benchmarks}

In this study, we focus on dynamics of biofilm growth with and without antimicrobial treatment. Thus, we choose two characteristic time scales corresponding to these two scenarios: the growth time scale at $t_{0}=10^{3}$ seconds without antimicrobial treatment and the time scale at $t_{0}=10$ seconds under antimicrobial treatment. We remark that the time scale for antimicrobial treatment can vary from minutes to hours, or even days depending on the choice of the antimicrobial agents and the outcome that one is interested in. We choose the smaller time scale comparable with the diffusion time scale of the antimicrobial agent in this study.

All the (dimensional) model parameters used in the current study are summarized in Table 1 with their respective references cited. In the following discussion, unless noted otherwise, the parameter values are chosen directly from Table 1 . Corresponding to the two characteristic time scales alluded to earlier, we will use two sets of dimensionless parameters in our simulations next.

To demonstrate the capability of the model, we first benchmark it against a known experiment [26], in which we use the experimental data on persisters and the total live bacteria in a developing biofilm to calibrate the model. The result is shown in Figure 2. Notice that there exists a lag phase in the biofilm experiment initially, which is supposedly regulated by growth factors [28, 37]. In our current model, this transient factor is not considered. So, we start the simulation at $t=2$, i.e. we bypass the delayed transient growth stage and apply our model to the later stage of biofilm growth. As shown in Figure 2, the model prediction agrees very well with the experiment in the time frame that it is supposedly valid [26].

In addition, we carry out another benchmark on spatially inhomogeneous biofilms to study dynamics of the biocide action against biofilms. In this experiment, some biofilm colonies are initially attached to the substrate; antimicrobial agents are then applied to treat the biofilm. The results are recorded in time series depicted in Figure 3. By taking the initial biofilm morphological profile from the experiment, our model with the parameters given in table I is employed to predict biofilm dynamics in the same time series. From figure 3, we observe the model predicted time sequences compare qualitatively well with the experimental time sequences reported in [16]. This lend us additional support for using the model to make dynamical predictions about biofilm dynamics. 
Table 1: Dimensional Paramters

\begin{tabular}{|c|c|c|c|c|}
\hline Symbol & Description & value & Unit & Source \\
\hline $\mathrm{T}$ & absolute temperature & 303 & Kelvin & {$[45]$} \\
\hline$k_{B}$ & Boltzmann constant & $1.38065 \times 10^{-23}$ & $m^{2} k g s^{-2} K^{-1}$ & {$[45]$} \\
\hline$\rho_{n}$ & biomass density & $1 \times 10^{3}$ & $k g m^{-3}$ & {$[45]$} \\
\hline$\rho_{s}$ & water density & $1 \times 10^{3}$ & $k g m^{-3}$ & {$[45]$} \\
\hline$h$ & characteristic length scale & $1 \times 10^{-3}$ & $\mathrm{~m}$ & {$[33]$} \\
\hline$t_{0}$ & characteristic time scale & 10 or 1000 & $\mathrm{~s}$ & {$[45]$} \\
\hline$L_{x}, L_{y}, L_{z}$ & size of computational domain & $1-2 \times 10^{-3}$ & $\mathrm{~m}$ & {$[45]$} \\
\hline$\eta_{b}, \eta_{p}$ & dynamic viscosity of bacteria and EPS & $2.7 \times 10^{2}$ & $k g m^{-1} s^{-1}$ & {$[27]$} \\
\hline$\eta_{s}$ & dynamic viscosity of solvent & $1.002 \times 10^{-3}$ & $k g m^{-1} s^{-1}$ & {$[27]$} \\
\hline$c_{0}$ & characteristic oxygen concentration & $8.24 \times 10^{-3}$ & $k g \mathrm{~m}^{-3}$ & {$[33]$} \\
\hline$d_{0}$ & characteristic antimicrobial concentration & $1.0 \times 10^{-2}$ & $k g m^{-3}$ & {$[43]$} \\
\hline$\gamma_{1}$ & distortional energy coefficient & $8 \times 10^{6}$ & $m^{-1}$ & {$[9]$} \\
\hline$\gamma_{2}$ & mixing free energy coefficient & $3 \times 10^{17}$ & $m^{-3}$ & {$[9]$} \\
\hline$\lambda$ & mobility parameter & $1 \times 10^{-9}$ & $k g^{-1} m^{3} s$ & {$[9]$} \\
\hline$\chi$ & Flory-Huggins parameter & 0.55 & & {$[45]$} \\
\hline$N$ & generalized polymerization parameter & $1 \times 10^{3}$ & & {$[45]$} \\
\hline$D_{c}$ & oxygen diffusion coefficient & $2.3 \times 10^{-9}$ & $m^{2} s^{-1}$ & {$[42]$} \\
\hline$D_{d}$ & antimicrobial diffusion coefficient & $6 \times 10^{-10}$ & $m^{2} s^{-1}$ & {$[43]$} \\
\hline$C_{2}$ & susceptible bacteria growth rate & $4 \times 10^{-4}$ & $s^{-1}$ & {$[11]$} \\
\hline$C_{3}$ & susceptible bacteria decaying rate & $4 \times 10^{-2}$ & $s^{-1}$ & {$[11]$} \\
\hline$r_{b s}$ & flush-out rate for susceptible & $4 \times 10^{-7}$ & $s^{-1}$ & estimated \\
\hline$r_{b d}$ & flush-out rate for dead bacteria & $1.0 \times 10^{-7}$ & $s^{-1}$ & estimated \\
\hline$b_{s p 1}$ & transfer rate & $2 \times 10^{-5}$ & $s^{-1}$ & estimated \\
\hline$b_{s p 2}$ & transfer rate & $1 \times 10^{-3}$ & $s^{-1}$ & estimated \\
\hline$b_{p s 0}$ & transfer rate from $\phi_{b s}$ to $\phi_{b p}$ & $4 \times 10^{-5}$ & $s^{-1}$ & {$[11]$} \\
\hline$C_{5}$ & EPS growth rate & $4 \times 10^{-4}$ & $s^{-1}$ & {$[13]$} \\
\hline$K_{1}, K_{3}$ & monod constant & $3.5 \times 10^{-4}$ & $\mathrm{~kg} \mathrm{~m}^{-3}$ & {$[33]$} \\
\hline$C_{7}$ & nutrient consumption rate & $4 \times 10^{-2}$ & $k g m^{-3} s^{-1}$ & {$[39]$} \\
\hline$C_{8}$ & antimicrobial agents consumption rate & $4 \times 10^{-2}$ & $k g m^{-3} s^{-1}$ & {$[39]$} \\
\hline$\phi_{b s 0}$ & carrying capacity for susceptible bacteria & 0.2 & & {$[36]$} \\
\hline$\phi_{b p 0}$ & carrying capacity for persister & 0.02 & & estimated \\
\hline$\phi_{p 0}$ & carrying capacity for EPS & 0.2 & & {$[18]$} \\
\hline
\end{tabular}

\subsection{Persister formation}

It is perceived that persister bacteria reproduce themselves in a low rate. Hence, the gain of persister bacteria in the biofilm is mainly due to the conversion from the susceptible cells under the condition of nutrient depletion or the existence of antimicrobial agents. Here, we conduct several numerical investigations to examine persister formation during biofilm development and to make comparisons with other existing conversion rate models, e.g., the constant conversion rate model and the nonlinear conversion rate model in equation (11).

One results on dynamics of biofilm development without antimicrobial treatment are shown in Figure 4. For a well-grown biofilm with depleted nutrient in the end, both the susceptible and the persister cells gradually diminish in the model of constant conversion rates and in the model of a nonlinear conversion rates in equation (11). These are in direct conflict with the experimental observations where persisters get into a dormant state [31] and can survive in a nutrient depleted environment $[32,7]$ at least for quite a long period of time. In comparison, our model for conversion rates agree qualitatively well with the survival persister population in the experiments in which the persister cell population decays only slightly over a long period of time.

We also conduct several 3D numerical simulations on biofilm development including persister formation in an infinite long tube and in a finite-length flow cell, respectively, taking into account the spatial inhomogeneity of the biofilm structure. In Figure 5, biofilm development in a quiescent aqueous environment is simulated, with nutrient supplied through the top surface $y=L_{y}$. Growth dynamics of several randomly distributed biofilm colonies developing into highly heteroge- 


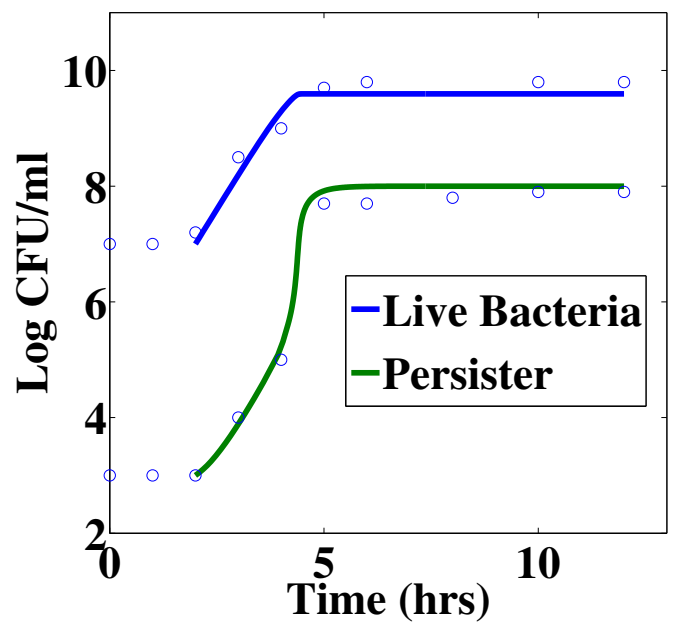

Figure 2: Benchmark of the model using experimental data of the total live bacteria and persisters. The curves are model predictions and circles are experimental data taken from [26]. The initial conditions are $\left(\phi_{b s}, \phi_{b p}, \phi_{d}, \phi_{p}, c, d\right)=\left(2 \times 10^{-4}, 2 \times 10^{-8}, 0,0,1.0,0\right)$. All parameters used are given in Table 1, except that $c_{2}=8 \times 10^{-4}, c_{7}=8 \times 10^{-3}$ and $\phi_{p 0}=0.002$. Here we assume the volume of each bacteria is about $2.71 \mu \mathrm{m}^{3}$, i.e, we use the initial bacterial volume fraction as $2 \times 10^{-4}$, which is equivalent to $10^{7} \mathrm{CFU} / \mathrm{ml}$ in the experimental measurement. We skip the initial transient lag phase in biofilm growth due to growth factors in the biofilm to focus our modeling on long time growth mechanisms. We note that our model is not developed to describe the initial lag phase.

nous biofilm colonies is shown in Figure $5(\mathrm{a}-\mathrm{e})$. To examine the distribution of each component internally, 2D slices at $x=0.5$ and $t=200$ are depicted in Figure 5(f-i). Notice that, in general, the susceptible cells are more concentrated near the region where nutrient is amply supplied. The volume fraction of the EPS pattern correlates with that of the susceptible cells as EPS is a product of live bacteria at the presence of nutrient. The persisters are primarily distributed inside the biofilm.

To further investigate the effect of nutrient distribution on persister formation in heterogenous environment, we carry out a comparative study with respect to four different nutrient concentrations supplied through the top surface. The results are shown in Figure 6. In any case, regardless if it is the nutrient sufficient or nutrient depleted case, persister cells would grow. However, in the nutrient depleted case, persister cells gain a higher ratio with respect to the total live bacteria than the case with more nutrient supplied. This is a direct consequence of low survival rate for susceptible cells in the biofilm. This perhaps explains why, in the nutrient depleted case, the biofilm is more persistent to antimicrobial treatment, as reported in some experiments $[1,2,3]$.

In Figure 7, we examine biofilm development in a flow cell with nutrient rich solvent flowing in through the inlet. The morphological change of biofilm colony is shown in Figure $7(\mathrm{a}-\mathrm{d})$ in the simulation. 2D slices at $x=0.5$ and $t=100$ are shown in Figure $7(\mathrm{e}-\mathrm{i})$. The migratory deformation in the colonies is clearly due to flow induced shear. The scenario for volume fractions of susceptible cells and EPS is similar to that depicted in Figure 5, i.e. they are more concentrated near nutrient rich regions within the biofilm. Persister cells are more concentrated inside the biofilm, where they have less access to nutrient.

In the above discussion, we focus primarily on growth dynamics of biofilms without the presence of antimicrobial agents. When the biofilm is treated by antimicrobial agents, some works have 

essential factor that has evaded modelers, that is the role of the nutrient concentration. As we have alluded to earlier, the conversion dynamics between persister and the susceptible cells relies heavily on the nutrient supply. Here, we carry out a comparative study using periodic dosing with respect to different nutrient conditions in the homogeneous model. In the following discussion, we set the nutrient concentration at a constant level, ignoring its dynamics in time. As shown in Figure 8, when the nutrient supply is high $(c=1)$, the live bacteria relapse, whereas if the nutrient supply is relatively low $(c=0.1)$, the biofilm is well-controlled. However, when the nutrient is low (i.e $c_{0}=0.01, c_{0}=0.025$ ), the volume fraction of persisters drops much slower. When nutrient is deficient at $c_{0}=0.0001$, the persister can hardly be treated. This study shows that the dependence of persister conversion on nutrient can affect antimicrobial treatment significantly. We hope this mechanism can be further confirmed by experimentalists so that we can come up with a better antimicrobial treatment strategy.

\subsection{Antimicrobial treatment of biofilms in a flow cell}

To develop a proper strategy for biofilm control and eradication, a clear understanding of antimicrobial dynamics in heterogeneous biofilms is important. We now investigate the antimicrobial process with respect to various antimicrobial dosing strategies in a long fluid channel as well as in a short flow cell.

In the first case, we examine antimicrobial treatment in a flow-cell, where the antimicrobial agents are carried into the cell through the inlet boundary by solvent. This mimics the antimicrobial treatment of biofilms in a pipe, by flowing disinfectant solutions. We depict the result in Figure 9. We observe that more susceptible bacteria are killed facing the inlet boundary than on the other side. Meanwhile, more susceptible bacteria in regions facing the incoming flow are converted into persister bacteria than those in the region facing the other way. This study also demonstrates the role played by antimicrobial agents in facilitating the conversion.

To show more details, $2 \mathrm{D}$ slices at $z=0.5$ are depicted in Figure $9(\mathrm{~g}-\mathrm{e})$. As one can observe in Figure 9(i), the distribution of the antimicrobial agents becomes heterogeneous within the biofilm, in particular, higher concentrations are observed near the inlet boundary. The consumption rate of antimicrobial agents is highly heterogeneous in the biofilm as well, shown in Figure $9(\mathrm{~g})$. This corroborates with the biofilm morphology depicted in Figure 9(d), where more persisters are converted from the susceptible cells due to the antimicrobial stress. We note that in the laminar flow field, there is a biomass flux moving towards the outlet boundary in the simulation (see Figure 9(j)) in which the disinfection process dominates. But, persisters can survive this treatment so that this dosing method fails to eradicate the biofilm colony satisfactorily.

With the development of nano-technology, new dosing strategies for biofilm have been investigated [25], in particular, targeted nano-scale encapsulated drug delivery for biofilms. In this context, we next conduct a study simulating targeted drug delivery in a flow cell, in which we investigate the combined effect of hydrodynamic environment and the interaction between biofilms and antimicrobial agents.

The first case we study is one single dose released in the flow cell adjacent to the biofilm subject to different inlet velocities (i.e., different hydrodynamic fluxes). The numerical results are shown in Figure 10. We observe that a higher inlet flow facilitates the antimicrobial effect on the biofilm so that more bacteria are killed initially. However, a faster flow also convects the antimicrobial agents out of the flow cell quicker so as to weaken the efficacy of antimicrobial agents in term of the overall antimicrobial effect. Figure 10(i) depicts the time-dependent concentration of antimicrobial agents in the flow cell on average. As the velocity increases, the antimicrobial agents are washed 
from the dead bacterial population in the cell shown in Figure 10(i).

Finally, we study the effect of dosing positions on antimicrobial efficacy, where we inject a single dose at several different positions relative to the biofilm. The numerical results are shown in Figure 11. The numerical study shows the second dosing position is more effective, where antimicrobial agents are released inside the biofilm to maximize contact with bacteria. In comparison, the first position is the least effective since the antimicrobial agents can easily flushed away by the flow before they even make contact with the bacteria. The dead bacteria for each dosing position are also shown in Figure 11. Overall, the targeted injection seems to be more effective as it kills more bacteria than the treatment by antimicrobial solutions. In particular, observed from Figure 11(c) and $11(\mathrm{~g})$, the antimicrobial agents are injected and exposed directly inside biofilms. It leads to an instantaneously concentrated disinfection. This is due to the fact that the reaction rate is much faster than the diffusion rate of the antimicrobial agents in this study.

\section{Conclusion}

In this paper, we develop a multiphasic field theory for biofilms taking into account interactions among multiple bacterial phenotypes, EPS, solvent, nutrient, and possibly antimicrobial agents. We classify the bacteria in biofilms into persistent cells and susceptible cells based on their response to antimicrobial treatment as well as dead cells. A new conversion rate model between the persistent and susceptible cells is implemented. A numerical scheme for solving the governing system of partial differential equations is developed and implemented on GPU in 3D space and time.

By focusing on a spatially homogeneous case, the model is calibrated against an experiment, which yields an excellent fit. The homogeneous model is then used to show its superiority over the two existing rate models in susceptible and persistent cell conversion when compared with experiments. In addition, the homogeneous model is also used to probe the effect of nutrient deficiency on the efficacy of antimicrobial treatment. Then, a series of $3 \mathrm{D}$ numerical simulations are carried out to investigate biofilm growth dynamics and the dynamical process of biofilm treatment by antimicrobial agents with respect to various dosing strategies. These studies reveal the crucial role played by the conversion mechanism between susceptible and persistent bacterial cells in biofilm dynamics with and without the antimicrobial treatment.

Experimentally, it is difficult to eradicate biofilms completely by conventional means. Thus, efforts in designing proper strategies are in need for biofilm control and eradication. The numerical simulations confirm that dosing by local release is much more effective as well as environmentfriendly than using a nebulizer, which delivers the antimicrobial agent to the surface of the biomasssolvent mixture or through an antimicrobial solution to rinse the biofilm. In particular, if antibiotics carrying nano-capsule can be delivered into the biofilm, the antimicrobial effect will be much more efficient, Besides dosing positions, dosing time controls are also vital for effective antimicrobial treatment and biofilm control, for which the model can also provide valuable insight.

\section{Acknowledgement}

Qi Wang is partially supported by AFOSR, NIH and NSF through awards FA9550-12-1-0178, DMS-1200487, DMS-1517347 and R01GM078994-05A1 as well as a SC EPSCOR GEAR award. Jia Zhao is partially supported by an ASPIRE grant from the Office of the Vice President for Research at the University of South Carolina. 


\section{References}

[1] S. M. Amato and M. P. Brynildsen. Nutrient transitions are a source of persiters in escherichia coli biofilms. PLOS One, 9(3):e93110, 2014.

[2] S. M. Amato, C. H. Fazen, T. C. Henery, and etc. The role of metabolism in bacterial persistence. Frontiers in Microbiology, 5(70):1-9, 2014.

[3] S. M. Amato, M. A. Orman, and M. P. Brynildsen. Metabolic control of persister formation in escherichia coli. Molecular Cell, 50(4):475-487, May 2013.

[4] N. Q. Balaban, J. Merrin, R. Chait, L. Kowalik, and S. Leibler. Bacterial persistence as a phenotypic switch. Science, 305(5690):1622-1625, August 2004.

[5] K. W. Bayles. The biological role of death and lysis in biofilm development. Nature Reviews Microbiology, 5(9):721-726, September 2007.

[6] N. Bell and M. Garland. Cusp: Generic parallel algorithms for sparse matrix and graph computations. preprint, 0:0, 2012.

[7] S. P. Bernier, D. Lebeaux, A. S. DeFrancesco, A. Valomon, G. Soubigou, J. Copppee, J. Ghigo, and C. Beloin. Starvation together with the sos response mediates high biofilm-specific tolerance to the fluoroquinolone ofloxacin. PLoS Genetics, 9(1), 2013.

[8] G. Borriello, L. Richards, G. D. Enrlich, and P. S. Stewart. Arginine or nitrate enhances antibiotic susceptibility of pseudomonas aeruginosa in biofilms. Antimicrobial Agents and Chemotherapy, 50(1):382-384, January 2006.

[9] C. Chen, M. Ren, A. Srinivansan, and Q. Wang. 3d numerical simulations of biofilm flows. East Asian Journal of Applied Mathematics, 1(3):197-214, 2011.

[10] H. Childs, E. Brugger, B. Whitlock, J. Meredith, S. Ahern, D. Pugmire, K. Biagas, M. Miller, C. Harrison, G. H. Weber, H. Krishnan, T. Fogal, A. Sanderson, C. Garth, E. W. Bethel, D. Camp, O. Rübel, M. Durant, J. M. Favre, and P. Navrátil. Visit: An end-user tool for visualizing and analyzing very large data. High Performance Visualization-Enabling ExtremeScale Scientific Insight, pages 357-372, 2012.

[11] N. G. Cogan. Effects of persister formation on bacterial response to dosing. Journal of Theoretical Biology, 238:694-703, February 2006.

[12] N. G. Cogan, Brown J, Darres K, and Petty K. Optimal control strategies for disinfection of bacterial populations with persister and susceptible dynamics. Antimicrobial Agents and Chemotherapy, 56(9):4816-4826, September 2012.

[13] N. G. Cogan and James P. Keener. The role of the biofilm matrix in structural development. Mathematical Medicine and Biology, 21(2):147-166, 2004.

[14] N. G. Cogan, B. Szomolay, and M. Dindos. Effects of periodic disinfection on persisters in a one-dimensional biofilm model. Bulletin of Mathematical Biology, 75:94-123, 2013.

[15] D. Davies. Understanding biofilm resistance to antibacterial agents. Nature Reviews Drug Discovery, 2:114-122, February 2003. 
[16] W. M. Davison, B. Pitts, and P. S. Stewart. Spatial and temporal patterns of biocide action against staphylococcus epidermidis biofilms. Antimicrobial Agents and Chemotherapy, 54(7):2920-2927, 2010.

[17] L. Demaret, J. H. Eberl, M. A. Efendiev, and R. Lasser. Analysis and simulation of a mesoscale model of diffusive resistance of bacterial biofilms to penetration of antibiotics. Advances in Mathematicsl Sciences and Applications, 18:269-304, 2008.

[18] R. M. Donlan. Biofilms microbial life on surfaces. Emerging Infectious Diseases, 8(9):881-890, September 2002.

[19] T. Dorr, M. Vulic, and K. Lewis. Ciprofloxacin causes persister formation by inducing the tisb toxin in escherichia coli. PLoS Biology, 8(2), 2010.

[20] Yang X. F. and Q. Wang. Energy stable numerical schemes for a hydrodynamic model of nematic liquid crystals. SIAM Journal on Scientific Computing, In press.

[21] O. Gefen and N. Q. Balaban. The importance of being persistent: heterogeneity of bacterial populations under antibiotic stress. FEMS Microbiology Reviews, 33(4):704-717, 2009.

[22] D. M. Grant and T. R. Bott. Biocide dosing strategies for biofilm control. Heat Transfer Enginnering, 26(1):44-50, 2005.

[23] K.J. Grobe, J. Zahller, and P.S. Stewart. Role of dose concentration in biocide efficacy against pseudomonas aeruginosa biofilms. Journal of Industrial Microbiology and Biotechnology, 29:1015, 2002.

[24] S. M. Hunt, M. A. Hamilton, and P.S. Stewart. A 3d model of antimicrobial action on biofilms. Water Science and Technology, 52(7):143-148, 2005.

[25] R. K Kasimanickam, A. Ranjan, G. V. Asokan, V. R Kasimanickam, and J. P Kastelic. Prevention and treatment of biofilms by hybrid and naotechnologies. International Journal of Nanomedicine, 8:2809-2819, 2013.

[26] I. Keren, N. Kaldalu, A. Spoering, Y. Wang, and K. Lewis. Persister cells and tolerance to antimicrobials. FEMS Microbiology Letters, 230:13-18, 2004.

[27] I. Klapper, C. J. Rupp, R. Cargo, B. Purvedorj, and P. Stoodley. Viscoelastic fluid description of bacterial biofilm material properties. Biotechnology and Bioengineering, 80(3):289-296, 2002 .

[28] N. Larsen, M. Boye, H. Siegumfeldt, and M. Jakobsen. Differential expression of proteins and genes in the lag phase of lactococcus lactis subsp lactis grown in synthetic medium and reconstituted skim milk. Applied and Environmental Microbiology, 72(2):1173-1179, February 2006.

[29] P. D. Leenheer and N. G. Cogan. Failure of antibiotic treatment in microbial populations. Journal of Mathematical Biology, 59(4):563-579, 2009.

[30] K. Lewis. Persister cells dormancy and infectious disease. Nature Reviews Microbiology, 5:48$56,2007$.

[31] K. Lewis. Persister cells. Annual Review of Microbiology, 64(1):357-372, 2010. 
[32] D. Nguyen, A. Joshi-Datar, F. Lepine, E. Bauerie, O. Olakanmi, K. Beer, G. McKay, R. Siehnel, J. Schafhauser, Y. Wang, B. Britigan, and P. Singh. Active starvation responses mediate antibiotic tolerance in biofilms and nutrient-limited bacteria. Science, 334:982-986, 2011.

[33] C. Picioreanu, M. C. M. van Loosdrecht, and J. J. Heijnen. Mathematical modeling of biofilm structure with a hybrid differential discrete cellular automaton approach. Biotechnology and Bioengineering, 58(1):101-116, April 1998.

[34] K. Poole. Bacterial stress responses as determinants of antimicrobial resistance. Journal of Antimicrobial Chemotherapy, 20(5):227-234, May 2012.

[35] J. H. Pyo and J. Shen. Gauge-uzawa methods for incompressible flows with variable density. Journal of Computational Physics, 221:181-197, 2007.

[36] M. E. Roberts and P. S. Stewart. Modeling protection from antimicrobial agents in biofilms through the formation of persister cells. Microbiology, 151:75-80, 2005.

[37] M. Rolfe, C. Rice, S. Lucchini, C. Pin, A. Thompson, A. Cameron, M. Alston, M. Stringer, R. Betrs, J. Baranyi, M. Peck, and J. Hinton. Lag phase is a distinct growth phase that prepares bacteria for exponential growth and involves transient metal accumulation. Journal of Bacteriology, 194(3):686-701, 2012.

[38] J. A. Sanclement, P. Webster, J. Thomas, and H. H. Ramadan. Bacterial biofilms in surgical specimens of patients with crhonic rhinosinusitis. Laryngoscope, 115(4):578-582, April 2005.

[39] P. Seeluangsawat. 3d computational investigation of viscoelastic biofilms using gpus. Ph.D. Thesis, 2011.

[40] J. Shen and X. Yang. Numerical approximation of Allen-Cahn and Cahn-Hilliard equations. Discrete and Continuous Dynamical Systems Series B, 28(4):1669-1691, 2010.

[41] M. Simões, L. C. Simões, and M. J. Vieira. A review of current and emergent biofilm control strategies. LWT - Food Science and Technology, 43(4):573 - 583, 2010.

[42] P. S. Stewart. Diffusion in biofilms. Journal of Bacteriology, 185(5):1485-1491, March 2003.

[43] Philip S. Stewart. Theoretical aspects of antibiotic diffusion into microbial biofilms. Antimicrobial Agents and Chemotherapy, 40(11):2517-22, 1996.

[44] B. Szomolay, I. Klapper, and M. Dindos. Analysis of adaptive response to dosing protocols for biofilm control. SIAM Journal of Applied Mathematics, 70(8):3175-3202, 2010.

[45] T. Zhang, N. G. Cogan, and Q. Wang. Phase-field models for biofilms i. theory and simulations. SIAM Journal of Applied Mathematics, 69:641-669, 2008.

[46] J. Zhao and Q. Wang. A 3d hydrodynamic model for cytokinesis of eukaryotic cells. Communications in Computational Physics, 19(3):663-681, 2016.

[47] J. Zhao and Q. Wang. Modeling cytokinesis of eukaryotic cells driven by the actomyosin contractile ring. International Journal for Numerical Methods in Biomedical Engineering, In press, 2016. 
[48] J. Zhao and Q. Wang. On energy stable schemes for a q tensor hydrodynamic model of liquid crystals. Journal of Scientific Computing, In press, 2016.

[49] J. Zhao, X. Yang, J. Shen, and Q. Wang. A decoupled energy stable scheme for a hydrodynamic phase field model of mixtures of nematic liquid crystals and viscous fluids. Journal of Computational Physics, 305:539-556, 2016. 


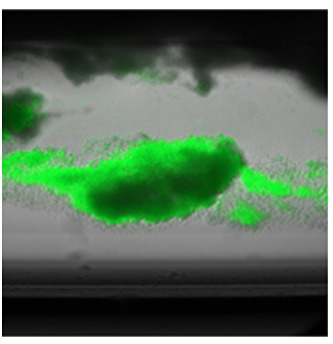

(a) $\mathrm{t}=0 \mathrm{~min}$

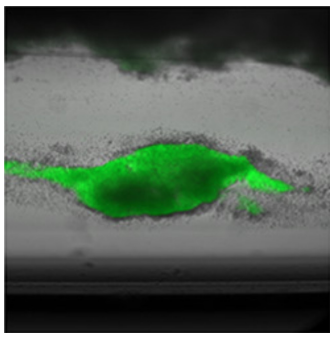

(b) $\mathrm{t}=10 \mathrm{~min}$

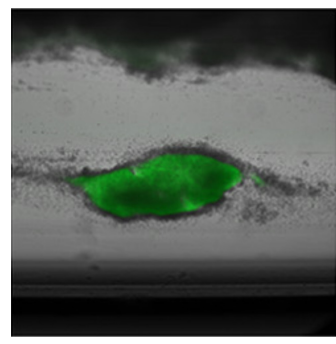

(c) $\mathrm{t}=20 \mathrm{~min}$

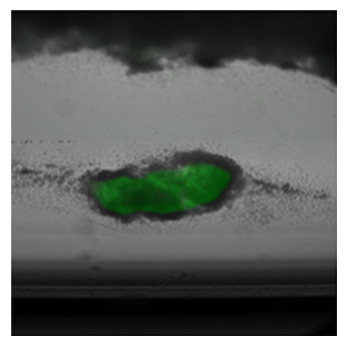

(d) $\mathrm{t}=30 \mathrm{~min}$

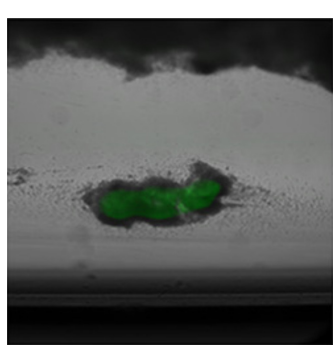

(e) $\mathrm{t}=40 \mathrm{~min}$

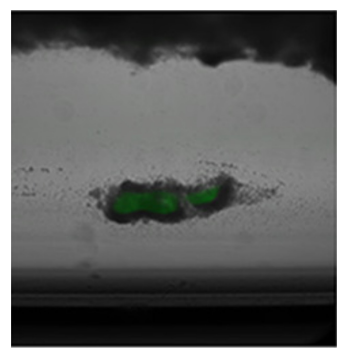

(f) $\mathrm{t}=50 \mathrm{~min}$

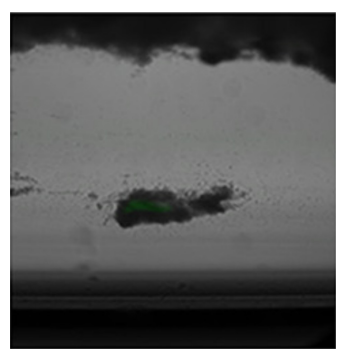

(g) $\mathrm{t}=60 \mathrm{~min}$

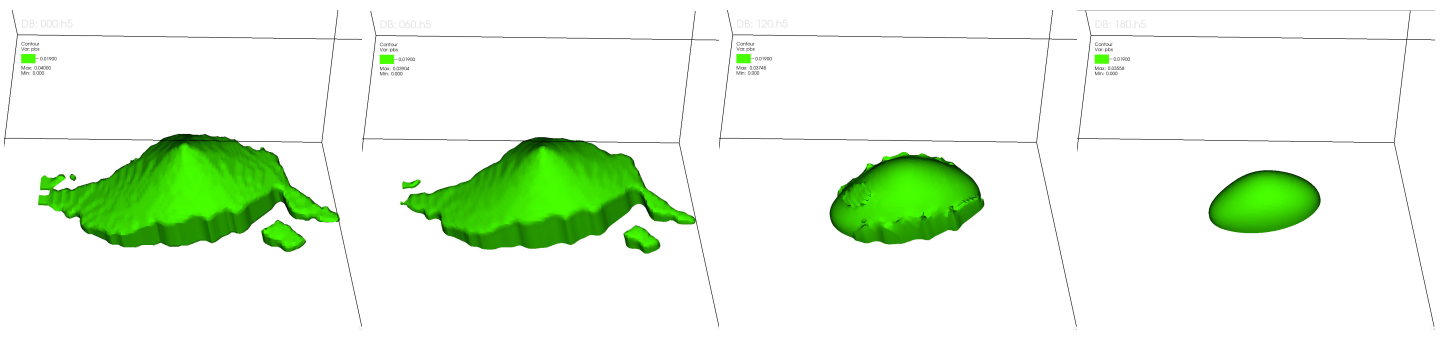
(h) $\mathrm{t}=0 \mathrm{~min}$
(i) $\mathrm{t}=10 \mathrm{~min}$
(j) $\mathrm{t}=20 \mathrm{~min}$
(k) $\mathrm{t}=30 \mathrm{~min}$

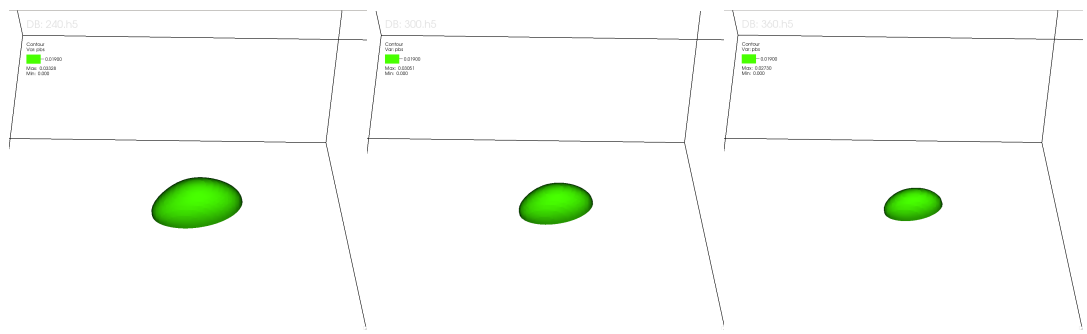
(l) $\mathrm{t}=40 \mathrm{~min}$
(m) $\mathrm{t}=50 \mathrm{~min}$
(n) $\mathrm{t}=60 \mathrm{~min}$

Figure 3: Antimicrobial treatment of biofilms. (a)-(g) Morphology of live bacteria aggregates (green) in S. epidermis biofilm at different time slots treated by $50 \mathrm{mg} / \mathrm{L} \mathrm{HOCl} \mathrm{[16].} \mathrm{(h-n)} \mathrm{numerical}$ predictions of live bacteria aggregates at the corresponding time slots treated by antimicrobial agents. All parameters used in the simulation are chosen from Table 1, except $C_{3}=4 \times 10^{-4}$. 
Figure 4: Comparison of biofilm growth dynamics in an environment with deficient nutrient supply using the homogeneous model. This figure shows that our new conversion rate model gives a better prediction for persister cells in the nutrient deficient case compared with the other existing models. (A). The volume fraction of susceptible cells. (B). The volume fraction of persistent cells. (C) The volume fraction of the dead bacteria. (D) The volume fraction of EPS. (E). The concentrate of nutrient. (F). The volume fraction of total live bacteria. In this case study, we examine an extreme case where the nutrient concentration is set to zero. The legend $X, Y, Z$ represent the conversion rate model of (27), constant rates and (11), respectively. 


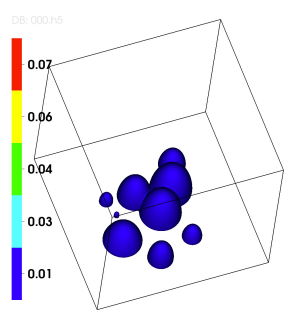

(a) $\phi_{n}$ at $t=0$

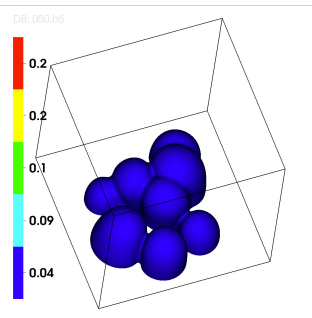

(b) $\phi_{n}$ at $t=50$

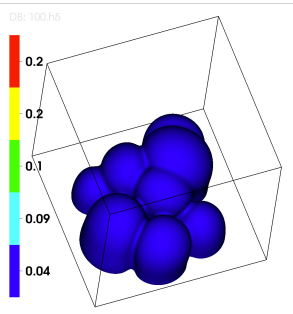

(c) $\phi_{n}$ at $t=100$

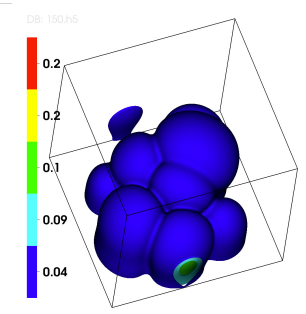

(d) $\phi_{n}$ at $t=150$

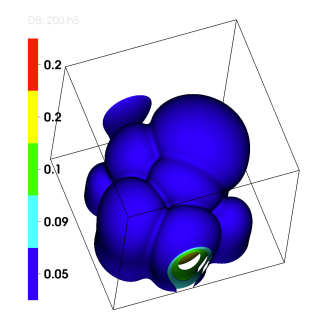

(e) $\phi_{n}$ at $t=200$

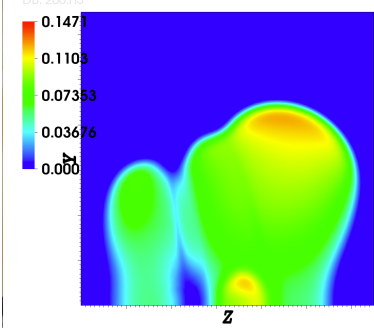

(f) $\phi_{b s}$ at $t=200$

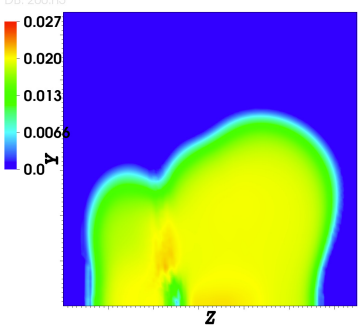

(g) $\phi_{b p}$ at $t=200$

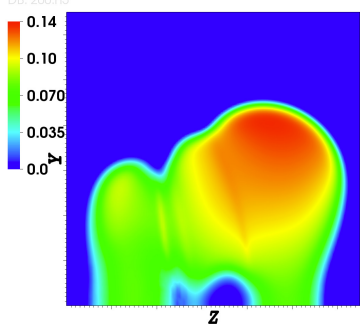

(h) $\phi_{p}$ at $t=200$

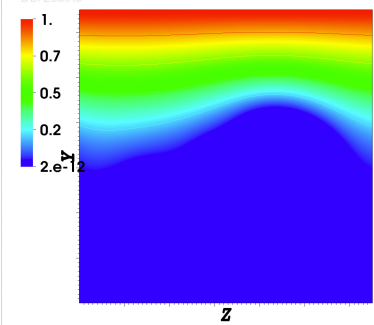

(i) $c$ at $t=200$

Figure 5: Persister formation in an infinitely long channel, where nutrient is supplied through the top boundary. The 3D view of biofilm colonies at different time slots are shown in (a-e). 2D slice view (at $x=0.5$ ) of the volume fraction of the susceptible cells, the persisters and EPS at $t=200$ are shown in (f-h), respectively. (i) The $2 \mathrm{D}$ slice view of nutrient concentration at $t=200$. 
Figure 6: Dynamics of persister growth. This demonstrates a comparative study on persister formation dynamics with respect to various nutrient supply conditions through the top boundary, namely, $c_{0}=1.0,0.5,0.1,0.01$ at $L_{y}$. The persister distribution at $t=200$ for the nutrient supply conditions are shown in (a-d), respectively. (e) The volume of total live cell, the susceptible cells and the persisters, i.e. $\int_{\Omega} \phi_{b s}+\phi_{b p} d \mathbf{x}, \int_{\Omega} \phi_{b s} d \mathbf{x}$ and $\int_{\Omega} \phi_{b p} d \mathbf{x}$, and the ratio of persister cells with respect to the total live bacteria, i.e. $\int_{\Omega} \phi_{b p} d \mathbf{x} / \int_{\Omega}\left(\phi_{b s}+\phi_{b p}\right) d \mathbf{x}$, are shown, respectively. $\Omega$ is the domain of the biofilm. The legend $1,2,3,4$ represent the simulations with $c_{0}=1.0,0.5,0.1,0.001$, respectively.

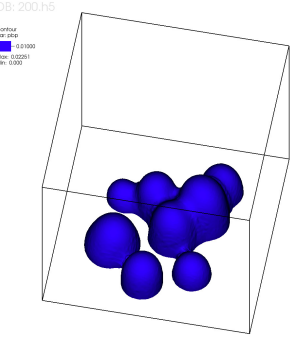

(c)

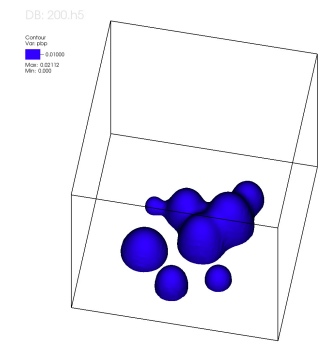

(d)

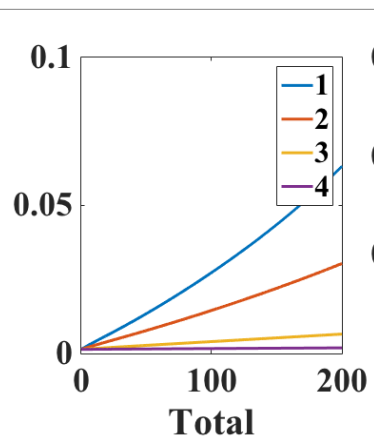

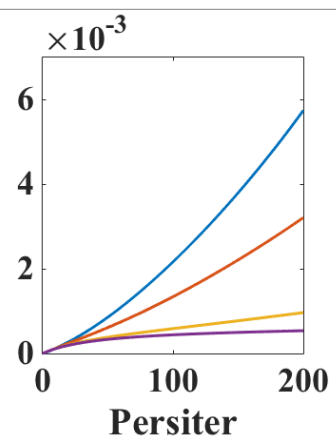

Persiter

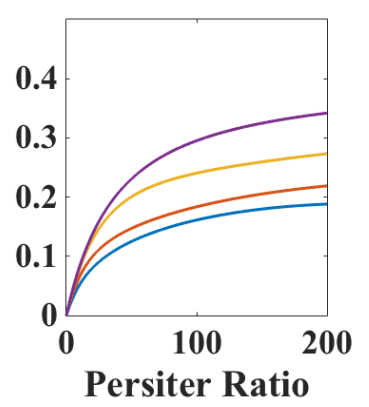

(e) 
Figure 7: Biofilm dynamics in a flow cell. This figure depicts the dynamical process of biofilm growth in a flow cell with fresh nutrient flowing through the cell.(a-d) show biofilm colonies at different time slots. (e-g) show $2 \mathrm{D}$ slices at $x=0.5$ of $\phi_{b s}, \phi_{b p}, \phi_{p}$ at $t=100$, respectively. The concentration of nutrient $c$ at $t=100$ is shown in (h). (i) The velocity field at $x=0.5$ and $t=100$. Flow induced deformation are observed.

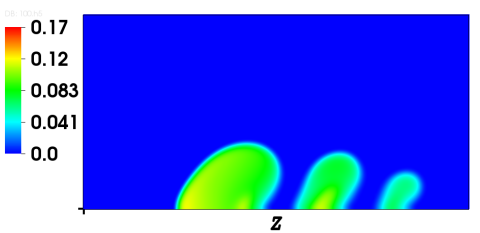

(e) $\phi_{b s}(x=0.5)$ at $t=100$

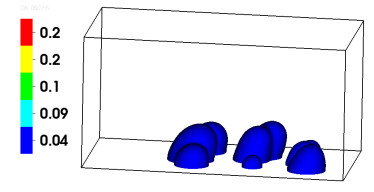

(c) $\phi_{n}$ at $t=50$

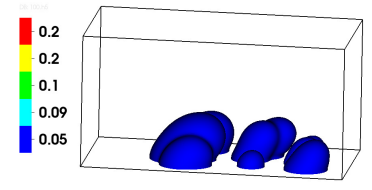

(d) $\phi_{n}$ at $t=100$

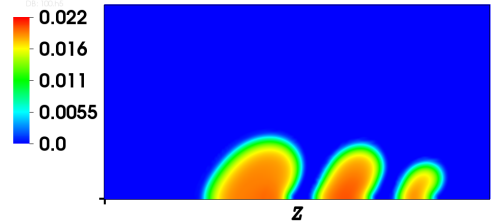

(f) $\phi_{b p}(x=0.5)$ at $t=100$

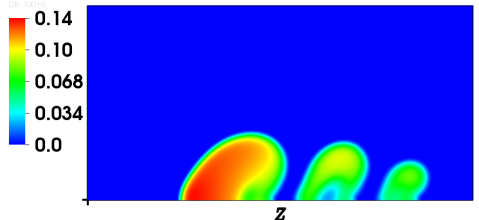

(g) $\phi_{p}(x=0.5)$ at $t=100$

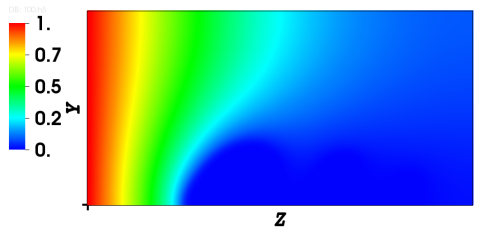

(h) $c(x=0.5)$ at $t=100$

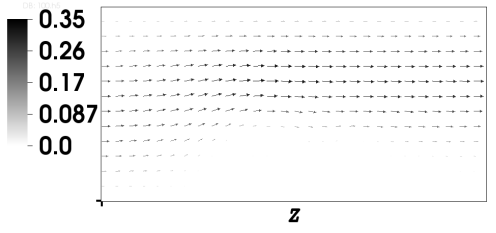

(i) $\mathbf{v}(x=0.5)$ at $t=100$ 

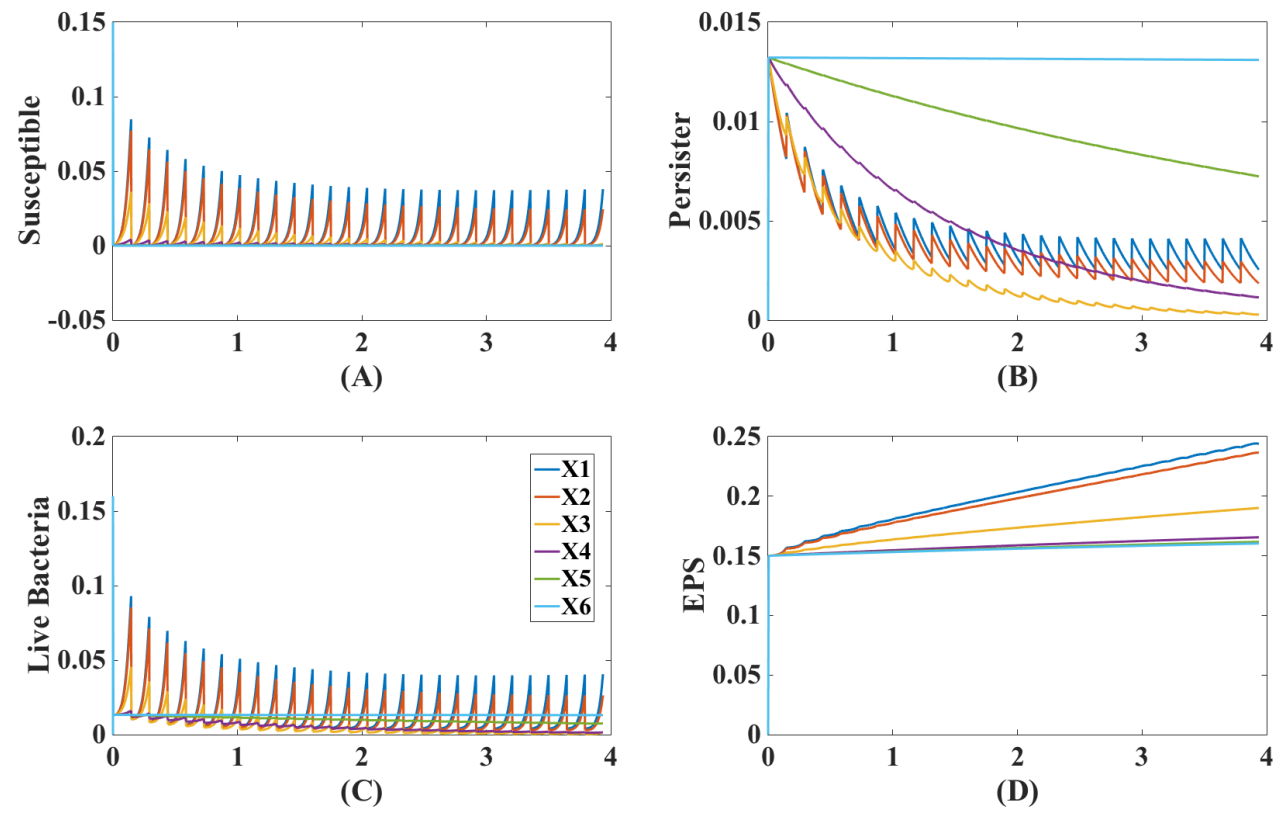

Figure 8: Dynamics of spatially homogeneous biofilms under antimicrobial treatment. This figure demonstrates how nutrient deficiency can reduce the efficiency of periodic dosing strategies. Here, we administer a periodic dosing with respect to various nutrient supply conditions: $c=1(X 1)$; $c=0.5(X 2) ; c=0.1(X 3) ; c=0.025(X 4), c=0.01(X 5), c=0.001(X 6)$. (A). The volume fraction of the susceptible cells. (B) The volume fraction of persisters. (C) The volume fraction of live bacteria. (D) The volume fraction of EPS. When the nutrient supply level is very low, the persister cells can hardly be treated. 


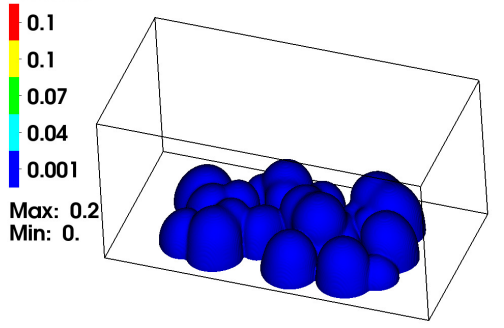

(a) $\phi_{b s}$ at $t=2$

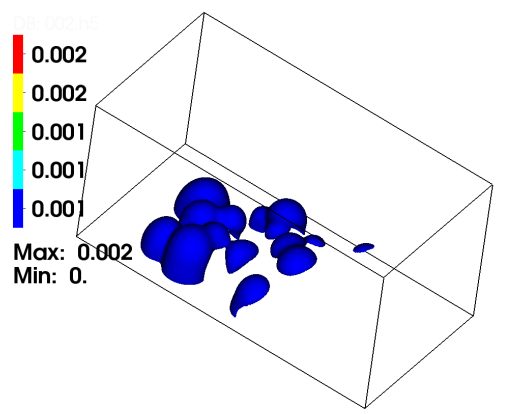

(d) $\phi_{b p}$ at $t=2$

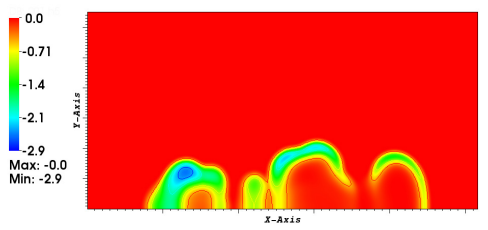

(g) $g_{d}$ at $t=2$

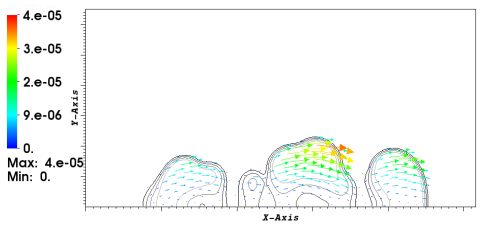

(j) $\phi_{n} \mathbf{v}_{n}$ at $t=2$

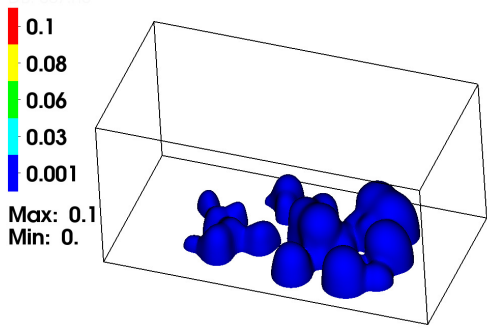

(b) $\phi_{b s}$ at $t=14$

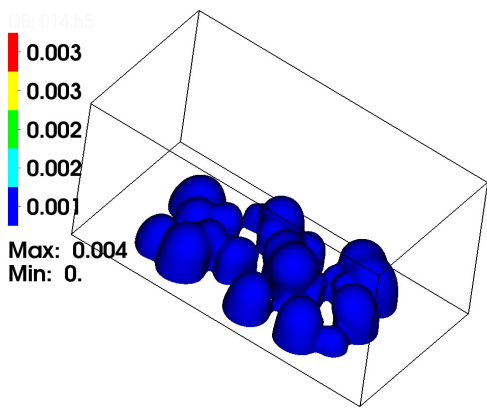

(e) $\phi_{b p}$ at $t=24$

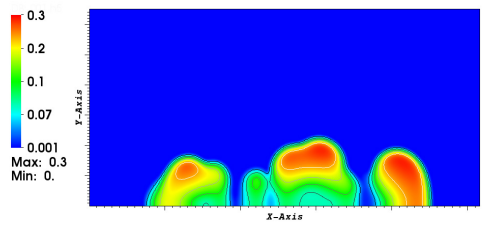

(h) Total Biomass at $t=2$

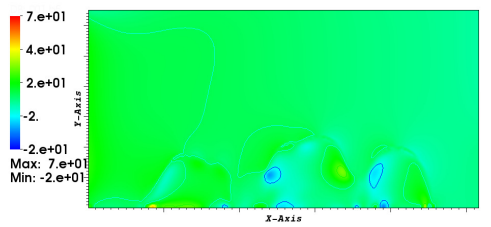

(k) Presure $p$ at $t=2$

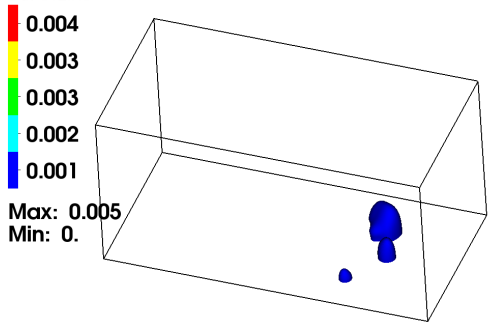

(c) $\phi_{b s}$ at $t=24$

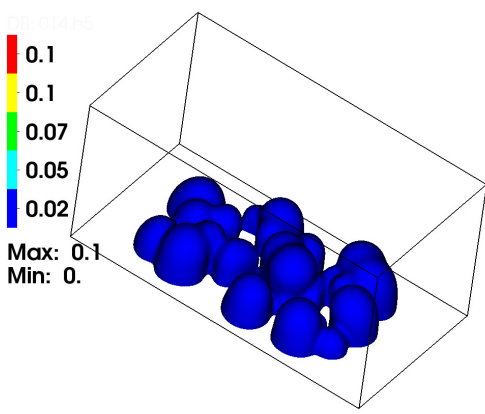

(f) $\phi_{b d}$ at $t=24$

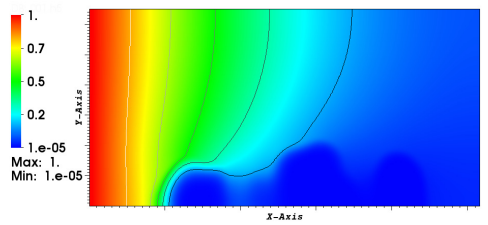

(i) $d$ at $t=2$

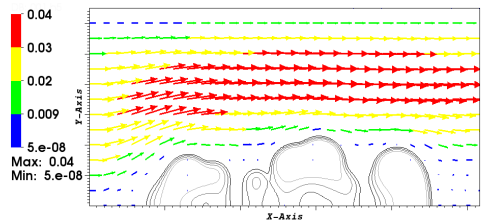

(l) $\mathbf{v}$ at $t=2$

Figure 9: Antimicrobial treatment of biofilms in a flow cell using antimicrobial solutions flowing through the inlet boundary. (a-c) The volume fraction of susceptible cells at time $t=2,14,24$, respectively. (d-e) The volume fraction of persisters at time $t=2,24$, respectively. (f) The volume fraction of dead bacteria at $t=24$. 2D slices at $z=0.5$ and time $t=2$ are shown in (g-l). (g) The consumption rate of antimicrobial agents. (h) The total biomass volume fraction. (i) The concentration of antimicrobial agents. (j) The biomass flux. (k) The hydrostatic pressure. There exists a few low pressure spots. But, they are not significant. (l) The velocity field. The flow above the biofilm is laminar. The treatment is effective on susceptible cells, but has little impact on persisters. 
Figure 10: Comparison of antimicrobial efficacy at various inflow velocities in a flow cell. (ad) show the concentration of antimicrobial agents at a few selected time slots at inflow velocity $\mathbf{v}_{0}=(1.0,0,0)$. (e-h) shows the volume fraction of dead bacteria at $t=15$ with respect to inlet horizontal velocities $v_{x 0}=1,2,4,8$, respectively. (i) shows the time series of the total mass of antimicrobial agents $\int_{\Omega} d d \mathbf{x}$ and total volume of dead bacteria $\int_{\Omega} \phi_{b d} d \mathbf{x}$ with respect to the four inlet velocities. $1,2,3,4$ correspond to $v_{x 0}=1,2,4,8$, respectively. The slower the inlet velocity is, the better antimicrobial treatment is. 
Figure 11: Comparison of antimicrobial efficacy at various dosing positions. The antimicrobial agents are released (or injected) at four distinct locations in a flow cell in which biofilms grow, $\mathbf{x}_{0}=(0.2,0.75,0.5),(0.45,0.2 ., 0.5),(0.2,0.2,0.5)$ and $(1.75,0.2,0.5)$, respectively. (a) The initial profile of the biofilm and velocity field; (b-e) The four dosing positions. (f-i) The volume fraction of dead bacteria at $t=15$ with respect to the four dosing positions. (j) The time series of the total mass of antimicrobial agents $\int_{\Omega} d d \mathbf{x}$ and the total volume of dead bacteria $\int_{\Omega} \phi_{b d} d \mathbf{x}$ for all four cases.

(g)

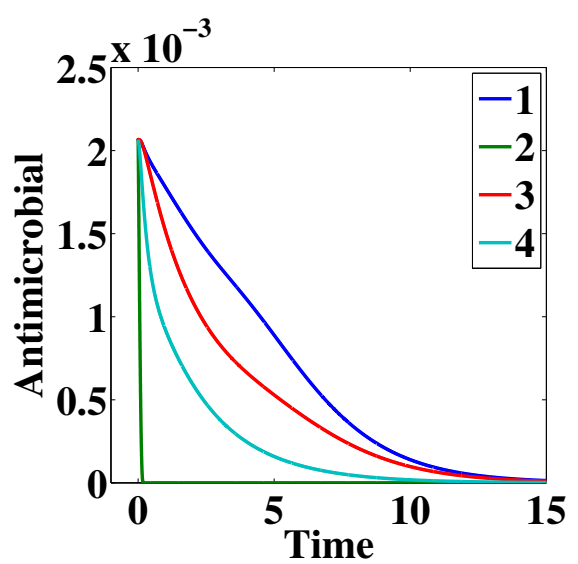

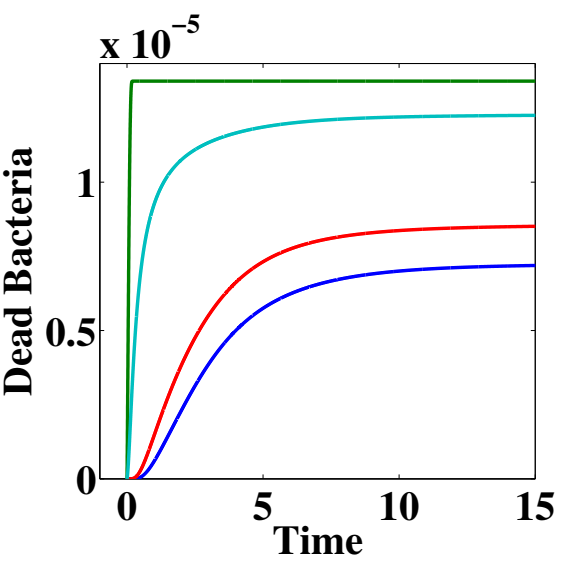

(j) (d)

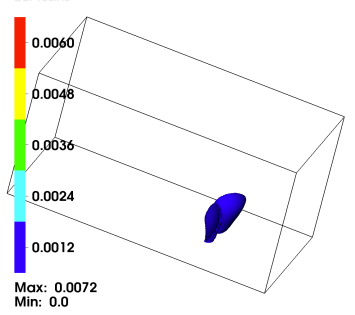

(i)

(e) 